Portland State University

PDXScholar

Summer 8-3-2017

\title{
Faculty Job Satisfaction and Morale in Biomedical Research
}

Richard John Goranflo III

Portland State University

Follow this and additional works at: https://pdxscholar.library.pdx.edu/open_access_etds

Part of the Educational Leadership Commons

Let us know how access to this document benefits you.

\section{Recommended Citation}

Goranflo III, Richard John, "Faculty Job Satisfaction and Morale in Biomedical Research" (2017).

Dissertations and Theses. Paper 3773.

https://doi.org/10.15760/etd.5657

This Dissertation is brought to you for free and open access. It has been accepted for inclusion in Dissertations and Theses by an authorized administrator of PDXScholar. Please contact us if we can make this document more accessible: pdxscholar@pdx.edu. 
Faculty Job Satisfaction and Morale in Biomedical Research

by

\title{
Richard John Goranflo III
}

A dissertation submitted in partial fulfillment of the requirements for the degree of

\section{Doctor of Education}

in

Educational Leadership: Postsecondary Education

\author{
Dissertation Committee: \\ Karen Haley, Chair \\ Candyce Reynolds \\ Kim D. Jones \\ Ellen West
}

Portland State University

2017 


\begin{abstract}
High faculty morale and job satisfaction are vital for optimum performance and important to the quality and vitality of the academic enterprise. However, research on faculty morale and job satisfaction has historically been limited to faculty at traditional comprehensive institutions and specific professional programs. Faculty who conduct biomedical research at academic health centers experience substantial differences in employment expectations and how they are funded than other faculty. The purpose of this study was to explore how personal and professional factors contribute toward positive morale and job satisfaction for faculty in biomedical research programs at one academic health center. This qualitative study used individual semi-structured interviews to explore work-life aspects associated with self-reported levels of morale and job satisfaction. Results from this study indicated that biomedical research faculty enjoy their work and highly value collaborating with their colleagues. The persistent need to fund at least half of their salaries through soft money, the loss of valued colleagues due to turnover, and a lack of identity with their institution decreases job satisfaction. It was also found that job satisfaction is expressed differently by gender and length of employment at one's current institution. Female faculty expressed feelings of limited support for those raising families while faculty employed longer expressed lower satisfaction than those recently hired. Better understanding of what influences job satisfaction and morale for this population will help academic health centers further support their research faculty as well as increase positive faculty identification with the institution.
\end{abstract}




\section{Table of Contents}

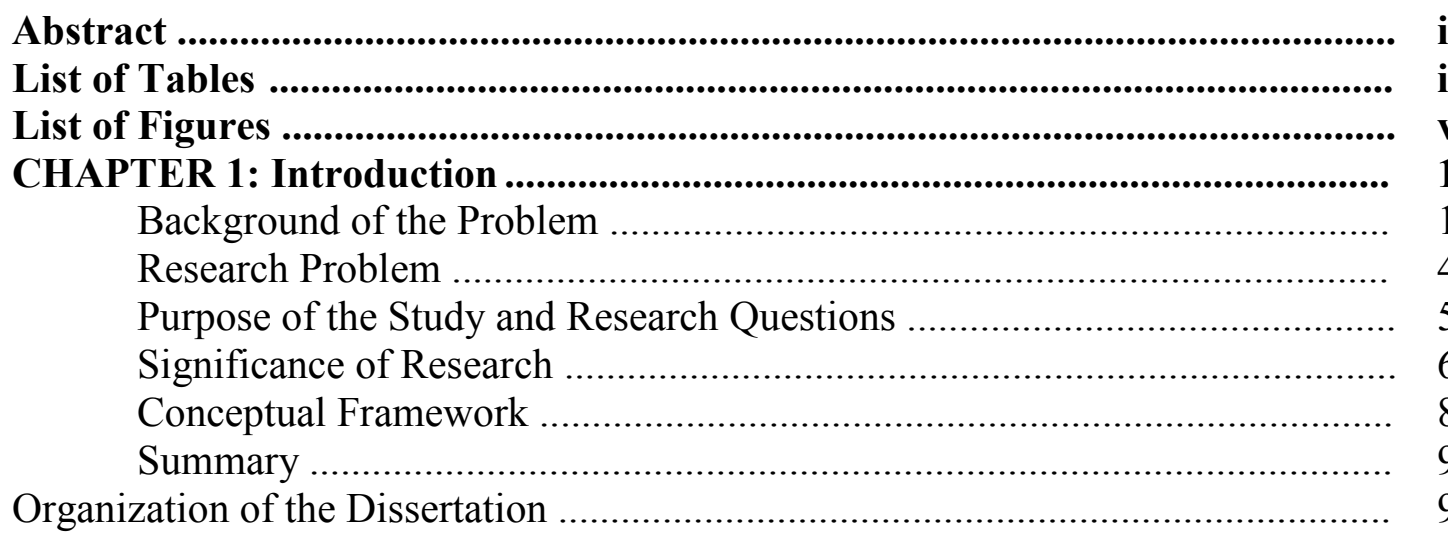

CHAPTER 2: Literature Review ................................................................................. 11

Morale \& Job Satisfaction ..................................................................... 11

Early Faculty Morale Research ............................................................. 12

Distinctive Organizational Cultures .............................................. 12

Participatory Leadership ............................................................. 13

Organizational Momentum ............................................................. 13

Faculty Identification with the Institution ........................................ 14

Conclusion ......................................................................... 14

Literature Review \& Conceptual Framework ........................................... 14

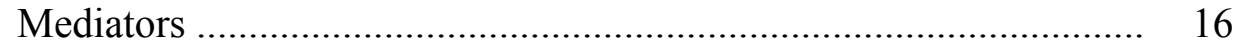

Triggers ……................................................................ 28

Job Satisfaction Continuum ........................................................ 32

Biomedical Research and Academic Medicine ............................................ 33

Funding Mechanisms ……………….......................................... 33

Faculty Wellness .......................................................................... 37

Academic Health Center Priorities .................................................. 38

Summary ................................................................... 39

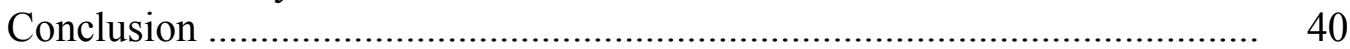

CHAPTER 3: Methodology ....................................................................................... 41

Methods ........................................................................................ 43

Participant Selection .................................................................. 43

Interview Protocol and Data Collection ......................................... 44

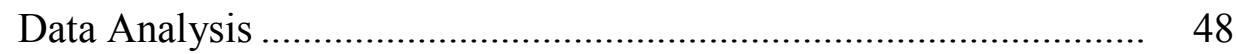

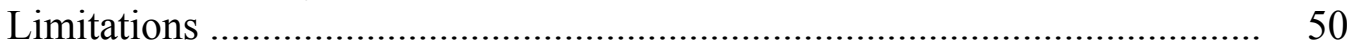

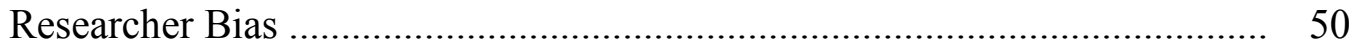

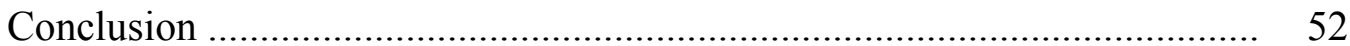

CHAPTER 4: Themes of Job Satisfaction ................................................................. 53

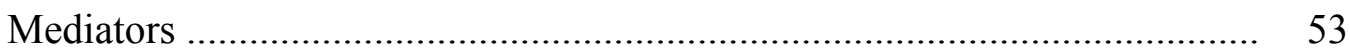

Motivators and Hygienes .............................................................. 53

Demographics ......................................................................... 65

Environmental Conditions ............................................................ 74 
Triggers ................................................................................... 85

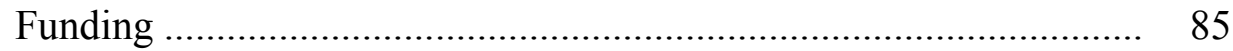

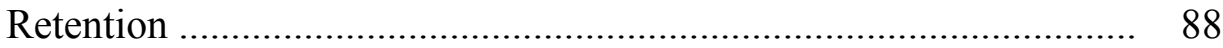

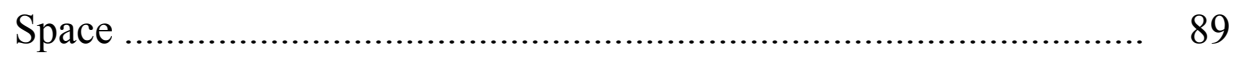

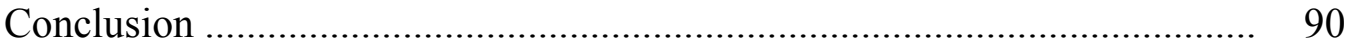

CHAPTER 5: Discussion and Implications ....................................................... 91

Conceptual Framework ...................................................................... 92

Research Question 1 ....................................................................... 93

Funding ............................................................................. 93

Collegial Relationships and Faculty Retention ........................... 95

Identification with the Institution ................................................. 96

Conducting Research and Work Itself ......................................... 97

Summary ............................................................................. 97

Research Question 2 .................................................................. 98

Female Faculty ................................................................. 98

Length of Employment ....................................................... 100

Summary .................................................................... 102

Recommendations for Academic Health Centers ..................................... 102

$\begin{array}{ll}\text { Talk to Faculty } & 102\end{array}$

Provide Funding Support ....................................................... 103

Enhance Retention Efforts .......................................................... 104

Increase Identification with Institution ........................................ 105

Support Female Faculty ......................................................... 106

Increase Faculty/Mission Alignment .......................................... 107

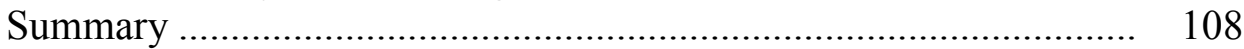

Future Research and Assessment ....................................................... 108

Assessment at Interview Site ..................................................... 109

Broader Surveying .................................................................. 109

Female Faculty ................................................................. 110

Soft Money Funding .................................................................... 111

Impact of Turnover on Remaining Faculty .................................... 111

Conclusion .................................................................................. 112

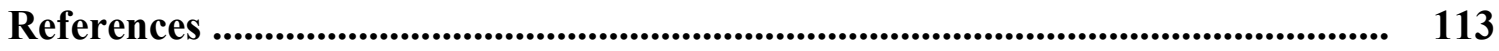

Appendix A: Introduction Email ..................................................................... 119

Appendix B: Informed Consent ................................................................................... 122

Appendix C: Interview Protocol ........................................................................ 125

Appendix D: Example of Coding ….......................................................................... 127 


\section{List of Tables}

Table 1: Participating Faculty by Gender and Length of Employment .........................45 


\section{List of Figures}

Figure 1: Conceptual Framework of Faculty Job Satisfaction ....................................15

Figure 2: Adapted Conceptual Framework of Faculty Job Satisfaction ........................ 92 


\section{CHAPTER 1: Introduction}

Although faculty have conducted biomedical research at academic health centers for decades, little effort has been made to understand what helps faculty in this specific discipline achieve high levels of job satisfaction and morale. While biomedical research faculty have always expressed high levels of stress, competition for funding, and long hours in the lab, the consequences of today's model of biomedical research funding has created an environment where scientists are becoming increasingly concerned for the wellbeing of their colleagues (Cole, Goodrich, \& Gritz, 2009; Holleman, Cofta-Woerpel, \& Gritz, 2014; Marburger, 2015). These concerns have escalated to the point where editorials about morale have been published in top tier journals such as Nature - arguably the most highly esteemed journal in biomedical research (Holleman \& Gritz, 2013). This study aims to better understand the primary concerns of faculty conducting biomedical research in academic health centers and to make recommendations as to strategies academic health centers can use to increase job satisfaction and morale.

\section{Background of the Problem}

"A grumbling faculty is a happy faculty. Even when the faculty get what they want...they still complain, because, that is what really makes them happy" (Flaherty, 2016, para. 25). While perhaps said in jest, this quote was posted on a popular higher education forum in response to an article about faculty outrage over a failed group hiring initiative at a research institution. This initiative impacted every department on campus, appears to have been made without faculty feeling their input was considered, and created a considerable amount of stress and distrust with the institution's faculty. This transcended mere complaining, but the perception that all complaining carries equal 
weight is misleading. Some complaints speak to issues directly related to morale and job satisfaction; issues which ultimately are "important to the quality and vitality of the academic enterprise” (Johnsrud \& Rosser, 2002, p. 538).

In recent years, universities have undergone a significant shift in how they are governed, funded, and regulated. These changes have gradually eroded aspects of the work environment that many faculty value. The perception of less participatory leadership, lower quality of benefits and services, and higher demand for administrative tasks are just a few factors that have ultimately contributed to a culture of low faculty morale (Holleman \& Gritz, 2013; Johnsrud \& Rosser, 2002).

Faculty who conduct biomedical research at academic health centers have seen additional changes that uniquely impact their work environment.

An academic health center encompasses all the health-related components of universities, including their health professions schools, patient care operations, and research. Thus, an academic health center consists of an allopathic or osteopathic medical school, one or more other health profession schools or programs (such as allied health, dentistry, graduate studies, nursing, pharmacy, public health, veterinary medicine), and one or more owned or affiliated teaching hospitals or health systems. (AAHC, 2014, para. 10)

These faculty often feel like second class citizens as they are usually responsible for obtaining their own funding, do not directly care for patients, and are typically compensated far less than their peers in clinical practice (Holleman et al., 2014; Holleman \& Gritz, 2013; Marburger, 2015). “Academic researchers function at the pressure point between the scientific importance of their work and its unstable future. 
Additionally, they must fund their research staff, protect sought-after lab space, publish steadily, teach, have national visibility, and provide both intramural and extramural service" (Cole et al., 2009, p. 5). With competition for limited funding at an all-time high (Cole et al., 2009; Marburger, 2015), it is reasonable to suspect that morale and job satisfaction would be negatively impacted.

Existing research on morale and job satisfaction often uses these terms interchangeably. While one could separate these into two distinct categories, this paper will keep in line with most morale and job satisfaction research by using the terms interchangeably. For this study, morale and job satisfaction are defined in this context as "the level of well-being that an individual or group is experiencing in reference to their work life" (Johnsrud \& Rosser, 2002, p. 524).

At its core, high morale is vital for optimum performance and " is important to the quality and vitality of the academic enterprise" (Johnsrud \& Rosser, 2002, p. 538). It has also been said that "if low job satisfaction or dissatisfaction exists amongst academics then the goals of higher education cannot be accomplished" (Eyupoglu \& Saner, 2009, p. 609). To foster an environment where faculty can meet or exceed institutional goals, it is important to continually monitor morale. Areas associated with low morale should be identified early, discussed before they diminish performance, and alleviated at a time when they are easier to address (Rice \& Austin, 1988).

Job satisfaction and morale can also differ depending on discipline, gender, race, academic rank, current health status, and marital status (Bender \& Heywood, 2006; Bilimoria et al., 2006; Bozeman \& Gaughan, 2011; Eyupoglu \& Saner, 2009; Fox \& Colatrella, 2006; Laden \& Hagedorn, 2000; Seifert \& Umbach, 2008; Wolf-Wendel \& 
Ward, 2014). While certainly helpful in assessing job satisfaction and morale in a specific work environment, what makes these criteria more complex to assess is that they often impact each other. For example, a female faculty member in a predominantly male department may present low levels of job satisfaction, while male faculty in a predominantly female department may not (Bender \& Heywood, 2006; Bilimoria et al., 2006; Wolf-Wendel \& Ward, 2014). Notably, non-married male faculty often report lower levels of job satisfaction and morale than non-married female faculty (Bender \& Heywood, 2006; Bilimoria et al., 2006; Callister, 2006). What is important is to acknowledge how these types of demographic indicators impact each other and influence the overall environment in a given department.

Most recently, researchers have begun to build on that knowledge by specifically distinguishing what factors impact faculty job satisfaction and morale by discipline. While there is existing research on faculty in general (Johnsrud \& Rosser, 2002; Rice \& Austin, 1988; Rosser, 2004), faculty at community colleges (Johnson, 2010), nursing faculty (Shockness, 2015), faculty as a whole in science, technology, engineering, and math (STEM) fields (Babin Verret, 2012), and even physicians at academic health centers (Cole et al., 2009; Nyquist, Hitchcock, \& Teherani, 2000), no study to date specifically looks at factors for biomedical research faculty at academic health centers.

\section{Research Problem}

Faculty in biomedical research programs report lower levels of job satisfaction, productivity, and personal physical and mental health in recent years (Cole et al., 2009; Holleman et al., 2014). However, it is currently unknown what influences morale and job satisfaction specifically for biomedical research faculty at academic health centers. 
Existing literature is mostly limited to faculty who teach undergraduates and very specific professional programs, which may not apply the same way to faculty who conduct biomedical research at very different institutions. Beyond concerns about funding availability to continue their research, little else is known about what components contribute to positive morale for biomedical research faculty.

While faculty have historically expressed some level of dissatisfaction with their jobs, in the context of newer trends such as increased responsibilities for accountability, more competition for federal funding, and increased overhead expenses on research grants, faculty feel especially pressured to find time to conduct research (Bender \& Heywood, 2006; Bilimoria et al., 2006; Marburger, 2015). Prior studies and national surveys have been conducted on broad issues that impact morale and job satisfaction for faculty, although none has been done to identify what most impacts faculty specifically in biomedical research. It is also unknown as to what relationships biomedical research faculty have that most impact their morale. Previous research has been done exclusively in the context of faculty relationships with their institutions, but little has looked at anything beyond that such as with their colleagues, the field the work in, or other communities.

\section{Purpose of the Study and Research Questions}

The purpose of this study is to explore the personal and professional factors that contribute to positive morale and job satisfaction for faculty in biomedical research programs at academic health centers. This study will explore factors related to how faculty navigate their organizations and institutional policies, their relationships with peers, issues of work life balance and self-care, job satisfaction, and their relationships 
within their professions. Obtaining this type of information about biomedical research faculty will provide a holistic approach to identifying how to increase morale and job satisfaction. The two research questions this study will address are:

1. How do biomedical research faculty at an academic health center describe the mediators and triggers that influence their morale and job satisfaction?

2. What are the differences in morale and job satisfaction of biomedical research faculty by gender and length of employment at their current academic health center?

\section{Significance of Research}

It is already known that faculty who are dissatisfied with their jobs are less productive, more likely to leave the institution, and display higher levels of absenteeism (Hagedorn, 2000; Smart, 1990). In that regard, it is crucial for any institution to make sure that their faculty operate at a high level of satisfaction with their jobs in order to maximize the university's resources. Likewise, it is in the best interest of funding agencies such as the National Institutes of Health to make sure that the scientists they fund are at peak productivity. Awarding research dollars to labs with low morale could mean lower scientific output and more difficulty in lobbying for future funding requests(Cole et al., 2009; Holleman et al., 2014; Marburger, 2015)

Overall health and wellness of faculty can also be impacted by low morale. Low morale can lead to an array of physical and mental health issues ranging from minor depression to suicidal ideation (Cole et al., 2009; Holleman \& Gritz, 2013). Biomedical research faculty have recently begun to form activities and groups focused on wellness in 
both formal and informal settings, but little is known about the larger problem of low satisfaction and where to focus institutional improvements.

If faculty job satisfaction were to continue at its perceived negative pace, one potential scenario might be fewer people conducting science, fewer people available to teach science, and a steep decline in federal research productivity. This impacts both individual institution as well as the health of the entire country's research enterprise. With that goes not just an abundance of knowledge, but an entire industry largely regarded as the most productive in the world (Marburger, 2015).

Learning more about this problem will benefit academic health centers who hire biomedical research faculty to contribute towards their academic mission. Understanding how to create an environment that enhances morale gives universities a better understanding how to support faculty who are more involved with educating students, more likely to provide service to the institution, and less likely to be absent (Rice \& Austin, 1988). Learning more about this topic can also potentially save academic health centers large sums of money due to less turnover of personnel. "The costs of faculty turnover are estimated to be $5 \%$ of a center's budget, not including the costs of lost opportunities, lost referrals, overload on remaining faculty, reduced productivity, and lower morale. Likewise, staff turnover is not only costly in itself, but also stresses faculty and disrupts their productivity" (Cole et al., 2009, p. 5).

Results from this study have the potential to help institutions evaluate their own environments and implement changes or solutions to improve biomedical research faculty morale. It is also intended for this study to be easily replicable at other institutions so 
they can evaluate their own unique faculty population and identify which factors are most important at their institution.

\section{Conceptual Framework}

A shortcoming often noted in faculty morale research is the lack of an appropriate single framework to gauge faculty morale. Instead, morale and job satisfaction research borrows from organizational and behavioral research to frame morale from a multitude of dimensions. While appropriate in some cases, Hagedorn's (2000) Conceptual Framework of Faculty Job Satisfaction pulls the strongest arguments from many different frameworks and summarizes them into one entirely focused on framing faculty morale and job satisfaction (Hagedorn, 2000).

Hagedorn's (2000) Conceptual Framework of Faculty Job Satisfaction divides factors that influence morale and job satisfaction into two segments; mediators and triggers. The first, mediators, are described as a "variable or a situation that influences (moderates) outcomes and relationships between other variables or situations producing an interaction effect" (Hagedorn, 2000, p. 6). Hagedorn sorts mediators into three distinct categories; motivators and hygienes, demographics, and environmental conditions.

The second component to Hagedorn's Conceptual Framework for Faculty Job Satisfaction is described as triggers. Triggers are described as the significant life events and circumstances that ebb and flow over time throughout one's life. While not always directly work-related, these events can positively or negatively impact ones morale or job satisfaction just as much as ones that do occur in the workplace (Hagedorn, 2000, p. 2).

This framework will be used to describe and support the literature around faculty morale and job satisfaction. This framework is also versatile enough to evaluate sub- 
populations of faculty within an institution, such as biomedical research faculty (Hagedorn, 2000).

\section{Summary}

Knowing what helps biomedical research faculty thrive at academic health centers may be leveraged towards creating healthier and more productive environments for biomedical research faculty everywhere. While issues regarding competition for funding are widely publicized, this study intends to look deeper into what impacts these faculty both positively and negatively and serves to create a conversation to address morale and job satisfaction of biomedical research faculty at academic health centers. This dissertation will explore existing literature pertaining to faculty job satisfaction, what an academic health center is, the qualitative study conducted, results from the study, and recommendations for future research.

\section{Organization of the Dissertation}

This paper is organized in five chapters. Chapter 2 explores existing literature related to the research problem. It describes early literature regarding faculty morale and job satisfaction, recent research describing differences in job satisfaction by various demographic and vocational characteristics, what an academic health center is, as well as a description of this study's conceptual framework.

Chapter 3 describes the methodology used in this study. This chapter describes the type of study conducted, the questions used in the study's interviews, who the participants are, and how data was analyzed. Chapter 3 also discloses limitations of the study as well as researcher bias.

Chapter 4 presents the study's findings. Responses from the study's interviews 
are sorted by the study's conceptual framework and describe which conditions increase or decrease faculty job satisfaction.

Finally, Chapter 5 concludes the study by providing answers to the study's research questions, implications for academic health centers, and recommendations for future research and assessment. 


\section{CHAPTER 2: Literature Review}

"If low job satisfaction or dissatisfaction exists amongst academics then the goals of higher education cannot be accomplished" (Eyupoglu \& Saner, 2009, p. 609). Ideally, every faculty member at every institution of higher education would express high levels of self-reported morale and job satisfaction. While that is unrealistic to expect, what can be done is explore what goes into creating an environment that fosters high job satisfaction and implement those best practices where appropriate.

This chapter presents research on factors that impact morale and job satisfaction in higher education. First, it introduces the foundations of morale and job satisfaction research from its early industrial beginnings to what has been established as the foundation of higher education job satisfaction research. Next, the conceptual framework used in this study is covered in detail with additional supporting research. Finally, it explores what is unique about biomedical research faculty at academic health centers and why their needs may differ from existing research on faculty morale and job satisfaction

\section{Morale \& Job Satisfaction}

Research on job satisfaction largely began from a human resources and organizational management mindset in the 1950s. Researchers, such as Frederick Herzberg, would often observe or interview factory workers to determine what kept them motivated, what impacted levels of productivity, and what retained good employees (Herzberg, Mausner, \& Snyderman, 1959). From there, research on job satisfaction branched out to more general office environments and many articles and dissertations have been published on the topic from various employment settings and viewpoints (Brief, 1998). Because higher education does not conform to the same conventions as a 
typical office environment, many of these practices are inappropriate or ineffective in maintaining high job satisfaction with university faculty. While a great starting place, future research has begun to take place specifically with faculty in higher education settings.

\section{Early Faculty Morale Research}

In 1988 Rice and Austin sought to identify what commonalities they could find in institutions with high self-reported morale of faculty. After surveying a subset of similar liberal arts institutions, four broad themes were found in institutions with the highest rates of reported faculty morale. Distinctive organizational cultures, participatory leadership, organizational momentum, and faculty identity with the institution they work at all contributed to faculty morale (Rice \& Austin, 1988). This preeminent study is the foundation on which much research on faculty morale is based and is worth specifically reviewing.

\section{Distinctive Organizational Cultures}

Noted as the most important single factor in determining faculty morale, institutions with distinctive organizational cultures or specific cultural missions rated the highest morale. Institutions in "survival mode" or continually chasing the next hot new major were perceived as having no vision. Aspects such as tradition, history, a notion of community, and even architecture were all part of what created a distinctive organizational culture (Rice \& Austin, 1988). Asking whether faculty feel like their institutions have a distinctive organizational culture and whether they feel their own personal goals resonate with that culture is especially important in assessing morale and job satisfaction. 


\section{Participatory Leadership}

Every institution that reported high levels of morale also had a high level of participatory leadership. Institutions with the highest morale seemed to fit a "strong leadership / flat hierarchy" (Rice \& Austin, 1988, p. 54) model of leadership. Faculty who felt they had influence in institutional decision making were happier. Conversely, those who felt marginalized by administrative leaders felt less engaged and reported lower morale. Participatory leadership manifests itself in every dimension of how a school functions, from what is taught in the classroom to engaging with trustees. One institution specifically noted in their institutional handbook, "the distinction between instruction and administration is meant to be only those of function and suggests no hierarchy of value related to the respective duties of each group" (Rice \& Austin, 1988, p. 55). Looking at organization charts as well as asking about feelings regarding institutional influence is critical in assessing faculty morale and job satisfaction.

\section{Organizational Momentum}

Faculty who identified with institutions who are "on the move" reported higher levels of morale. Organizational momentum could involve having faced adversity and then moving past it, but the common theme was institutions that were going somewhere, paving the way for something new, and faculty were on board for that journey.

Organizational momentum can manifest itself in any level of an institution; whether from the very top or within a small department or office. The important concept is that faculty felt like they were a part of something moving the institution forward (Rice \& Austin, 1988). This may differ across an institution in a variety of settings, so specifying a distinct population to assess is important in gauging organizational momentum. 


\section{Faculty Identification with the Institution}

Finally, Austin \& Rice (1988) found that faculty with strong identification with their institutions reported higher levels of morale. Factors such as collaboration, focused support, a sense of "home," and clear priorities all factor into institutional identification (Rice \& Austin, 1988). At its core, those who best "fit" with the institution were the happiest. This can manifest itself in many different ways, but primarily those with the strongest institutional identity also reported individual goals and commitments similar to the institution. This also extended to the university's surrounding area and the community in which is resided. This too can differ across campus or even within departments, but assessing overall feelings of identification with the institution within a distinct population of faculty is important in assessing morale and job satisfaction.

\section{Conclusion}

Almost 30 years later, Rice and Austin's 1988 work is still the foundation on which most faculty morale research is built. Distinctive organizational cultures, participatory leadership, organizational momentum, and faculty identification with the institution are all critical in assessing morale and job satisfaction of faculty at any given institution. All four categories will be explored in this study in regards to how they impact morale and job satisfaction in biomedical research faculty.

\section{Literature Review \& Conceptual Framework}

A shortcoming often noted in faculty morale research is the lack of an appropriate single framework to gauge faculty morale. Instead, morale and job satisfaction research borrows from organizational and behavioral research to frame morale from a multitude of dimensions. While appropriate in some cases, Hagedorn's (2000) Conceptual 
Framework of Faculty Job Satisfaction pulls the strongest arguments from many different frameworks and summarizes them into one entirely focused on framing faculty morale and job satisfaction. This framework (see Figure 1) will be used to describe the literature around faculty morale and job satisfaction and how the framework supports that literature. This framework is also versatile enough to evaluate sub-populations of faculty within an institution (Hagedorn, 2000).

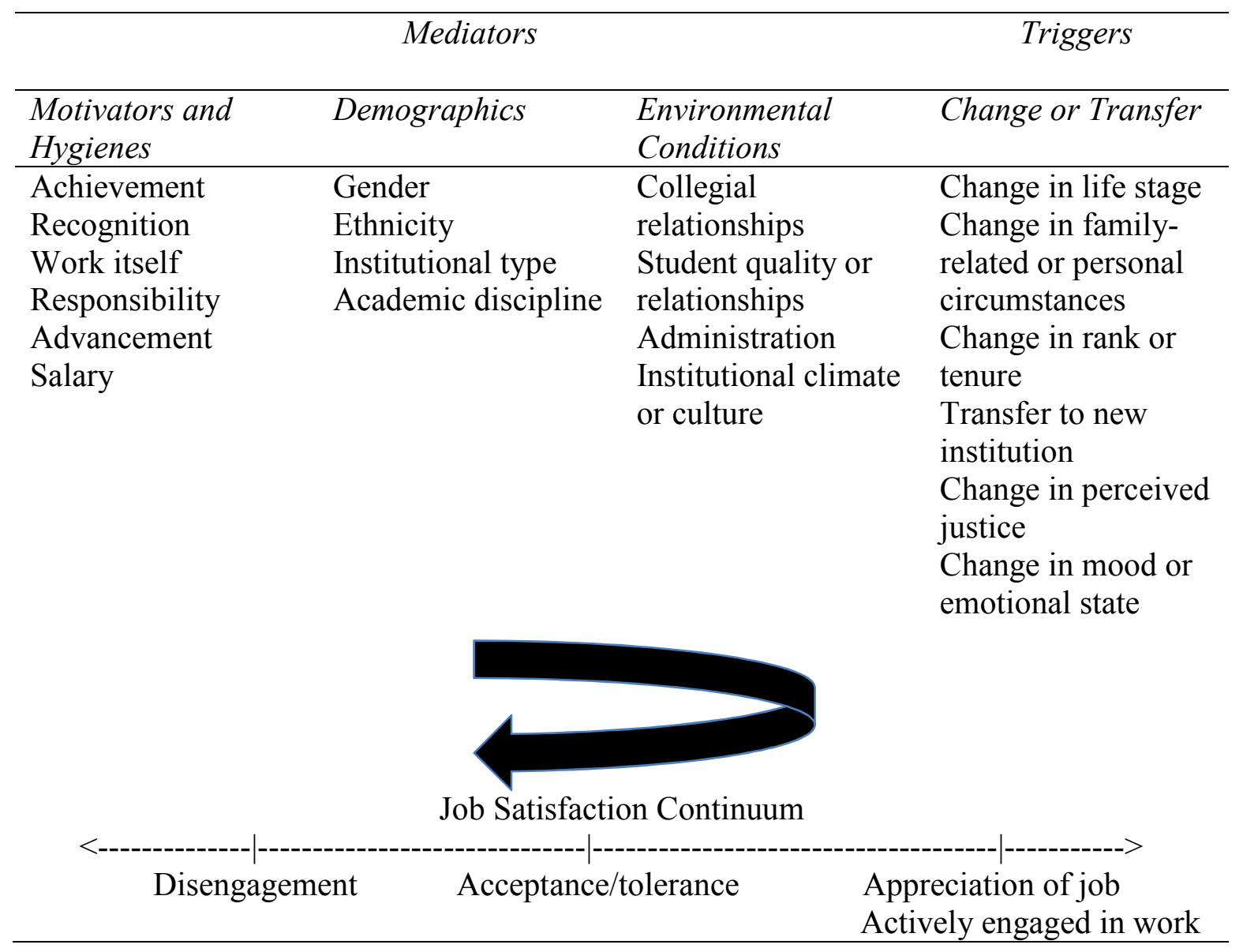

Figure 1. Hagedorn's (2000) conceptual framework of faculty job satisfaction by Hagedorn, L. S., 2000, Conceptualizing faculty job satisfaction: Components, theories, and outcomes. In L. S. Hagedorn (Ed.), What contributes to job satisfaction among faculty and staff, p. 7. Copyright 2009 by Jossey-Bass. 


\section{Mediators}

Hagedorn's (2000) Conceptual Framework of Faculty Job Satisfaction divides factors that influence morale and job satisfaction into two segments; mediators and triggers. The first, mediators, are described as a "variable or a situation that influences (moderates) outcomes and relationships between other variables or situations producing an interaction effect" (Hagedorn, 2000, p. 6). These variables uniquely impact job satisfaction and morale for each individual and there is no "one size fits all at all times" (Hagedorn, 2000, p. 6) approach to measuring which are good and which are bad. It is understood that the variables constantly influence each other and can be situationally negative or positive and are fluid in that what contributes positively in one situation may contribute negatively in another or at a later stage in life. Hagedorn sorts mediators into three distinct categories; motivators and hygienes, demographics, and environmental conditions.

Motivators and hygienes. Motivators and hygienes are based on early job satisfaction research conducted in the 1950s and 1960s by Herzberg and his colleagues (as cited in Hagedorn, 2000) at the time to describe which factors increase worklife satisfaction (motivators) and which decrease satisfaction (hygienes). Motivators and hygienes are typically directly related to one's work, such as feelings of achievement, feeling adequately recognized for one's achievements, the work itself, responsibility and autonomy, advancement, and (to a lesser degree) salary. "Thus, when a worker feels a high level of achievement, is intensely involved, and is appropriately compensated by recognition, responsibility, and salary, job satisfaction is enhanced and job dissatisfaction 
is decreased" (Hagedorn, 2000, p. 8) While originally developed to describe job satisfaction in a more general business-like environment, motivators and hygienes can also be applied to faculty in academia. These same issues frequently come to light in faculty surveys as well as key points in union-represented negotiations (Brannen et al., 2014; Foster \& Lyman, 2012; TWU, 2015). When assessing for faculty morale and job satisfaction, it will be important to separate issues related to motivators and those associated with hygienes.

Demographics. Basic demographics such as gender and ethnicity can impact one's perception of job satisfaction both positively and negatively. Hagedorn (2000) also includes academic discipline and institutional type under demographics as both are inherent to a faculty member's identity as a teacher and scholar. For example, a female faculty member in an otherwise all-male department may report lower levels of satisfaction due to perceived pressures of additional responsibilities to represent their department in various capacities as the only female faculty member. The same holds true for faculty who may be the only person of color or representative of any number of underrepresented demographics (Laden \& Hagedorn, 2000; Ropers-Huilman, 2000; Wolf-Wendel \& Ward, 2014). That feeling of being the only person of a certain demographic can come with dissatisfaction independent of the work itself.

Gender. In assessing morale and job satisfaction, it is important to examine differences in how faculty members experience their work environment by gender. Although Hagedorn (2000) specifically notes gender in a neutral fashion, almost all research related to differences in worklife experience by gender is focused on how women experience academia differently than their male counterparts. Equally important 
to note is that those different experiences are not necessarily negative and are sometimes dependent on other variables such as discipline, age, or marital status. Issues ranging from families to tenure to collegiality all play an important role, but no absolute consensus currently exists (Bender \& Heywood, 2006; Fox \& Colatrella, 2006; Sabharwal \& Elizabeth, 2009; Seifert \& Umbach, 2008; Wolf-Wendel \& Ward, 2014).

Women and academic discipline. While there are few generalizations when considering faculty job satisfaction, one largely agreed upon outcome from the research is that female faculty express lower job satisfaction in disciplines that are typically dominated by male faculty (e.g., engineering, computer science, math, and economics). Women also leave academic positions in science and engineering at higher rates than men (Callister, 2006). In comparison, women typically report higher levels of job satisfaction and morale in social science and professional healthcare disciplines where higher levels of female faculty are more common (Bender \& Heywood, 2006; Callister, 2006; Fox \& Colatrella, 2006; Sabharwal \& Elizabeth, 2009; Seifert \& Umbach, 2008; Wolf-Wendel \& Ward, 2014).

What is also clear is that the dissatisfaction does not stem simply from having to work with men, but rather in those departments who assess performance by standards set by men (Bender \& Heywood, 2006; Sabharwal \& Elizabeth, 2009). Female faculty working in male-dominated fields are often asked to perform additional advising or service tasks that men are not, which can potentially take them off track from achieving tenure and promotion goals (Sabharwal \& Elizabeth, 2009; Wolf-Wendel \& Ward, 2014). Female faculty can fall short of formal promotion and tenure goals because of these additional tasks that are often not weighed heavily in promotion criteria. For example, 
certain committees (such as elected positions) may count towards promotion $\&$ tenure, whereas others (such as appointed positions) may not. The dichotomy of this type of work environment creates a lose-lose scenario that usually decreases job satisfaction (Bender \& Heywood, 2006).

One stark difference in how female faculty reported feeling treated in academia is around the issue of parenthood. Female faculty expressed perceived additional pressures regarding parenthood that male faculty do not (Fox \& Colatrella, 2006; Sabharwal \& Elizabeth, 2009; Seifert \& Umbach, 2008). Some disciplines go so far as to lead women to believe that those who are serious about their careers should forego becoming mothers all together. That pressure is felt even greater in professional fields where women feel they must "opt out" of motherhood in order to be taken seriously and advance through tenure (Wolf-Wendel \& Ward, 2014).

In one study, Wolf-Wendel and Ward (2014) interviewed 118 female faculty members who identified as new mothers from an even distribution of institutional types and disciplines across the United States. These interviews were conducted in two phases approximately eight years apart in order to collect initial perceptions as brand new mothers and eight years later to determine whether those perceptions persisted or not. Results were then divided by discipline type to determine which fields felt what types of pressures.

Ultimately, Wolf-Wendel and Ward (2014) found that there were indeed differences in perception by academic mothers between different disciplines. While many women early in their careers (phase one) and mid-career (phase two) expressed similar concerns, women in science, technology, engineering, and math (STEM) and 
professional (law, medicine, education) fields felt much more tension between their career trajectory and their family life than those did in the humanities (Wolf-Wendel \& Ward, 2014). This finding is consistent among similar studies surveying female faculty across disciplines (Bender \& Heywood, 2006; Callister, 2006; Fox \& Colatrella, 2006; Sabharwal \& Elizabeth, 2009; Seifert \& Umbach, 2008).

One notable difference is how research is conducted in their fields. In the humanities, faculty members expressed a greater sense of independence where they conduct much of their research on their own and can do so on their own time. Those in STEM fields, however, often participate in team-based research where one might be in charge of running a laboratory setting. One scientist noted that due to labs essentially running $24 / 7$, one does not have the luxury of simply taking time off without potentially losing all of one's competitiveness for grant funding.

In other disciplinary areas, there was ebb and flow to research productivity, but the STEM faculty maintained more continuity and research productivity. We attribute this finding to the need to stay current, maintain labs through grant funding, and also the group orientation of lab spaces. (Wolf-Wendel \& Ward, 2014 , p. 24)

While initially not their intention, Wolf-Wendel and Ward (2014) also discovered that many women in STEM and professional fields often feel like they are the only one. As sometimes the only female faculty member in their program or department, many felt like they had to serve on committees, take on additional advising loads, and disproportionately represent their department at university or community functions.

A biologist at a comprehensive college told us how she is constantly getting 
involved in presidential initiatives, the most recent one related to a cross-campus initiative to support interdisciplinary research. As the only woman in her area and the only person with grant-writing experience, she is regularly called upon to participate in this type of work. (Wolf-Wendel \& Ward, 2014, p. 23)

These additional pressures can put burdens on female faculty that can take away from research, time with their families, or other interests that would lead to a more wellrounded work life balance. This finding is also consistent with similar studies regarding feeling isolated in the workplace (Callister, 2006; Fox \& Colatrella, 2006; RopersHuilman, 2000).

In summary, identifying the difference in experiences between male and female faculty is important in assessing morale and job satisfaction in faculty. If female faculty feel they are working under different expectations than their male counterparts, those differences should be identified and considered for future opportunities to change how a department or school sets expectations for its faculty.

Gender and leadership. Although women are slowly beginning to gain more leadership roles in higher education, women are still underrepresented at the highest levels. While numbers of female graduates are rising in traditionally underrepresented programs such as those in science, technology, engineering, and math (STEM) fields, those who make up current faculty are typically far less representational. As such, many of the customs, norms, and expectations of faculty are derived from conditions set by men (Bateh, 2013; Bilimoria et al., 2006; Kezar \& Lester, 2008).

Literature also demonstrates that many higher education environments have not fully embraced women teachers, learners, and leaders. In particular, women with 
progressive or feminist commitments, because they often work against the norms of university life in some ways, struggle to gain full acceptance in the academic groves. (Ropers-Huilman, 2000, p. 21)

When a woman does become a leader, one significant issue is that she is unlikely to enact her preferred leadership model due to either organizational structure or environmental issues (Kezar \& Lester, 2008). Traditionally, organizational roles have been top-down and authoritative with little collaboration due to strict hierarchies. This model is in contrast to how many women leaders report as their preferred methods of creating strong inter-professional relationships and participatory governance. If an organizational model or the culture of an institution does not allow for one's preferred leadership style, it has been found that women leaders will often speak of their preferred leadership styles, but exhibit the traditional leadership style of the men before them (Kezar \& Lester, 2008). Breaking this cycle of upholding male-dominated leadership expectations will take time, but latest best practices in leadership are beginning to recommend the exact types of methods women leaders have reported as preferential (Kezar, 2014).

Race and ethnicity. Race and ethnicity also play a role in how faculty experience morale and job satisfaction. For faculty of color at an institution of higher education, it is not uncommon for them to find that they are the only person in their department who looks like them (Antonio, 2003; Johnsrud \& Rosser, 2002; Laden \& Hagedorn, 2000; Seifert \& Umbach, 2008). With that feeling of isolation can come a number of other issues impacting job satisfaction; such as lack of emotional support, perceived expectations to serve on more committees, and an expectation to mentor students of a similar background (Laden \& Hagedorn, 2000). Compared to their white counterparts, 
faculty of color are also less likely to be represented in higher academic ranks, are typically less satisfied with every aspect of their jobs, exhibit more stress through the promotion and tenure process, and more likely to report discrimination in the workplace (Antonio, 2003; Laden \& Hagedorn, 2000; Seifert \& Umbach, 2008).

For those who decide to stay in academia under these conditions, it has been found that job satisfaction and morale are often times derived differently for faculty of color. At times, those activities that increase job satisfaction in one way can be detrimental in others. For example, "the longer road to tenure may in part be due to the large blocks of time faculty of color report spending in advising and mentoring students of color, serving on multiple institutional committees (many which have ties to diversity), or participating in community services" (Laden \& Hagedorn, 2000, p. 60). Even if a faculty member of color enjoys these activities, they are largely undervalued or not considered at all in evaluation of promotion and tenure.

Similarly, "another issue of discomfort is that all too often faculty of color find themselves placed in positions of being expected to handle minority affairs and speak as the minority expert" (Laden \& Hagedorn, 2000, p. 62). This puts faculty of color in a particularly tough situation. If they do not participate in activities related to diversity, they may miss out on opportunities to enact real cultural change on their campus. If they do, they then expend more time on activities not directly related to how they are evaluated for career progression.

Ultimately, faculty of color typically express satisfaction through three main variables: (1) satisfaction with teaching and working with students; (2) supportive administrative leadership, mentoring relationships, and (3) collegiality and interaction 
with other faculty (Laden \& Hagedorn, 2000). In other words, the same variables that largely motivate faculty of all kinds are the same that motivate faculty of color (Antonio, 2003; Laden \& Hagedorn, 2000; Seifert \& Umbach, 2008). To best achieve high morale and job satisfaction, it is recommended institutions become cognizant of the additional perceived workloads on their faculty of color and provide supportive environments for them to thrive.

Environmental conditions. The third mediator in Hagedorn's model is to consider the environmental conditions of one's workplace. While broad in nature and somewhat subjective, an institution's climate and culture are important to consider when evaluating morale and job satisfaction (Hagedorn, 2000). For example, a campus where faculty feel that their voices are not heard may lead to a climate where faculty feel unimportant or powerless over their environments. Conversely, one where faculty feel their voice is heard and are part of processes they feel are important can lead to a climate that increases satisfaction (Bateh, 2013; Bilimoria et al., 2006; Bozeman \& Gaughan, 2011; Johnsrud \& Rosser, 2002; Rice \& Austin, 1988).

Related to environmental conditions is one's ability to form collegial relationships. A number of factors such as campus size, organization, and location can impact this, but the broader concept is whether or not a campus encourages collaboration with other faculty. One campus environment where this may not be possible, for example, is one in which faculty are expected to fiercely compete with each other for resources. Faculty may feel it is against their best interests to get to know each other as it may result in potential feelings of sabotage or accidentally giving away information that could be used against them. An environment where faculty are physically separated from 
each other by long distances can have a similar effect over time. When opportunities to collaborate with colleagues are few, feelings of isolation will decrease satisfaction overtime (Bozeman \& Gaughan, 2011; Hagedorn, 2000; Sabharwal \& Elizabeth, 2009). The following are environmental conditions described by Hagedorn (2000) and supported by related literature.

Professional priorities and rewards. "Faculty members indicate that their greatest sources of satisfaction include the degree of autonomy they enjoy in their work and the intellectual challenge of their jobs" (Johnsrud \& Rosser, 2002, p. 522). What professional priorities and rewards do not include is salary or other types of monetary rewards. Instead, one of the leading causes of stress for faculty is attempting to accomplish everything they need to do in a certain amount of time and with competing priorities. The more their own professional priorities match those of their institution or department, the greater job satisfaction and morale they typically express (Rice \& Austin, 1988).

One competing demand in more recent times is the quantification of faculty work and the national push for accountability in higher education. "Recent attacks from the public and state legislatures are twofold: they demand workload studies to determine not only how hard or how much faculty members work, but also to question those tasks to which they devote their time" (Johnsrud \& Rosser, 2002, p. 522). Johnsrud and Rosser have determined that the relatively recent addition of public demands for faculty accountability have added another source of faculty stress. Many of those demands are centered on undergraduate education, which for faculty at research institutions may not be a significant portion of their job. Accountability measures in other areas, such as 
mandatory reporting needs for research grants or other federal funding mechanisms, also diminish faculty job satisfaction and increase stress (Marburger, 2015). Institutions that can best manage public demands and integrate them appropriately into institutional priorities will show higher levels of faculty satisfaction (Johnsrud \& Rosser, 2002). While just one example, any competing priority has the potential to diminish job satisfaction if considered strongly negative.

Administrative relations and support. This concept refers to the extent to which faculty feel their leaders are advocating on behalf of the faculty and the confidence they have in their decision-making. While almost identical to the concept of participatory leadership, Johnsrud \& Rosser (2002) also describe instances in which faculty involvement in decision-making is not ideal and how having faculty-minded leaders are an asset. For instance, "faculty governance processes are notoriously slow and cumbersome" (Johnsrud \& Rosser, 2002, p. 523). In times of immediate crisis, there may be instances in which getting a group consensus is impossible or inappropriate.

"Interestingly, there is evidence that faculty participation in budget decisions actually increases during financial crises, but nonetheless, morale drops" (Johnsrud \& Rosser, 2002, p. 524). In these cases, having leaders who have the best interests of faculty in mind would be beneficial for both quick and faculty-appropriate decision making.

Quality of benefits and services. While salary has never been identified as a primary contributor to morale or job satisfaction, faculty largely report as not being satisfied with their current levels of income. Beyond income, "other support services, such as access to research support, clerical support, graduate assistants, computing and technological support, even parking, are also important to the quality of worklife" 
(Johnsrud \& Rosser, 2002, p. 524). Most important regarding benefits and support services is perceived equity in how they are distributed. Differences between colleges, departments, or other sub-groups can significantly decrease morale (Johnsrud \& Rosser, 2002). Recent examples of this have come to light when institutions are forced to consolidate; especially when with two dissimilar institutions are merged (Brannen et al., 2014). Regardless of the issue, it is important to assess perceived satisfaction of benefits and services when taking into account the overall satisfaction of a group of faculty.

Organizational leadership. Factors known to impact faculty morale are often a product of how institutions are organized and governed (Cohen \& March, 2000; Holleman et al., 2014; Johnsrud \& Rosser, 2002; Kezar \& Lester, 2008; Marion \& Gonzales, 2014; Rice \& Austin, 1988). Because of this, it is important to understand the dynamics of organizational leadership and how it ultimately impacts faculty morale. Exploring organizational leadership at an institution through organizational theories and lenses provides insights into solutions or future best practices.

Surveys of faculty morale often indicate that faculty have a negative perception of bureaucracy (Brannen et al., 2014; Foster \& Lyman, 2012; TWU, 2015). Those surveys also indicate that faculty are not interested in navigating bureaucracy and often regard it as a barrier. Knowing how to navigate it can lead to positive outcomes, while not understanding it can lead to frustration or dissatisfaction. Therefore, knowing how to navigate an institution's bureaucracy is necessary in changing policies or procedures that impact faculty morale (Mintzberg, 2000). It may be that the organizational leaders perceived as barriers to higher morale are those that can help make changes. Knowing what is important to organizational leaders can also help frame recommendations that 
benefit their work or initiatives as well.

Summary. Motivators and hygienes span a wide breadth of conditions and experiences that faculty can perceive uniquely. While it would be nearly impossible to create a perfect environment for every unique faculty member, knowing that their experience can be different depending on a number of demographic, gender, and environmental conditions is important in ultimately understanding which elements of an environment most positively (motivators) and negatively (hygienes) impact it. When identified, an institution or department can begin working on individual issues instead of the entire morale picture at once (Laden \& Hagedorn, 2000).

\section{Triggers}

The second component of Hagedorn's Conceptual Framework for Faculty Job Satisfaction is described as triggers. Triggers are described as the significant life events and circumstances that ebb and flow over time throughout one's life. While not always directly work-related, these events can positively or negatively impact ones morale or job satisfaction just as much as ones that do occur in the workplace. Some universities have positioned themselves to assist with these types of life events by offering services such as counseling, wellness offerings, or work schedule or tenure adjustments for various reasons. "Despite brave (and undoubtedly costly) efforts by the institution, triggers (such as divorce, death of a loved one, birth, and so on) will pull faculty and staff attention away from the job. Perhaps compassion is what is more doable" (Hagedorn, 2000, p. 2). The following are the six triggers Hagedorn (2000) outlines as especially related to faculty job satisfaction.

Change in life stage. "Because life and work are intertwined, the transition into 
life stages plays a prominent role in job-related outcome. For example, Baldwin proposed a tri-stage theory of the faculty career consisting of (1) early career, (2) midcareer, and (3) late career" (as cited in Hagedorn, 2000, p. 10) What motivates or drives a faculty member at one stage in their career may become a deterrent in another. For example, faculty in their early career may be more interested in startup funds or the tenure process, whereas faculty in their late career may deeply care about an institution's policies around retirement. Faculty will naturally progress through these stages and what is most important for an institution to recognize is that support for all stages are needed in order to maintain high satisfaction across campus (Bender \& Heywood, 2006; Eyupoglu \& Saner, 2009).

Change in family-related or personal circumstances. Life's ebbs and flows naturally bring with it changes in family-related or personal circumstances. Instances such as birth, death, divorce, illness of self or significant other are part of everybody's shared experience in academia.

Researchers in the area of work-family conflict have found that conflict between the job and family concerns provide stress that ultimately can affect both psychological and physical health. This trigger is highly interactive with gender as the conflict is generally more acute for females. (Hagedorn, 2000, p. 11) This trigger is further supported by previously mentioned research regarding job satisfaction and family life (Bender \& Heywood, 2006; Bilimoria et al., 2006; Sabharwal \& Elizabeth, 2009; Seifert \& Umbach, 2008; Wolf-Wendel \& Ward, 2014)

What is important is to have policies and practices in place to help one's employees cope with these by feeling supported in the workplace. Policies such as 
flexible time off, adjustable tenure clocks for faculty with new families, and robust health insurance benefits are all examples of how one's personal circumstances can be benefitted even a little bit by support in the workplace (Hagedorn, 2000; Sabharwal \& Elizabeth, 2009; Wolf-Wendel \& Ward, 2014).

Change in rank or tenure. As faculty perceptions change through different life stages, so too do they change as they progress through rank and tenure.

A change in rank brings a new outlook on the position, different expectations, and a change in responsibility. More specifically... assistant professors dwell on advancing in the profession; at the associate level the focus switches to the establishment of balance within professional life; and finally, full professors can define their professional life and fulfill their lifetime goals. (Hagedorn, 2000, p. 11)

Promotions can elicit the excitement of new responsibilities as well as the stress and weight that come with them. Institutions with clear tenure guidelines and supportive senior faculty to mentor junior faculty are likely to be most successful in changing of rank or tenure to be a positive trigger.

The impact of tenure on job satisfaction is perhaps the most widely agreed upon factor that has been studied thus far. In short, the higher rank one has, the more likely they are to express higher levels of morale and job satisfaction. Full professors tend to report the highest levels of satisfaction while untenured faculty report the lowest across faculty of all disciplines (Bender \& Heywood, 2006; Bozeman \& Gaughan, 2011; Eyupoglu \& Saner, 2009; Hagedorn, 2000; Sabharwal \& Elizabeth, 2009). While tenure and academic rank increase job satisfaction and morale to both male and female faculty, 
the reported increase in job satisfaction is typically higher for male faculty than it is female faculty (Bender \& Heywood, 2006; Sabharwal \& Elizabeth, 2009). Knowing this, assessing morale and job satisfaction by rank is important in fully understanding a population of faculty as those of different ranks will likely have different concerns.

Transfer to a new institution. Faculty tend to be rather mobile as they progress through their careers. Instead of staying at one institution and rising through its tenure process for one's entire career, many faculty find it in their best interest (both financially and professionally) to transfer to new institutions.

Regardless of the reasons why a faculty member moves to a different institution, the change in locale will always mean new surroundings, responsibilities, students, colleagues, and fitting oneself within a different institutional mission. Thus, like other triggers, a change in institution results in movement on the continuum. (Hagedorn, 2000, p. 12)

Changes in institution also often bring forth a change in family status for those who may have to relocate with a spouse and/or children. Acclimating one's entire family to a new environment can bring with it an additional sense of stress in the workplace.

Change in perceived justice. Perceptions of justice or fairness play an important role in morale and job satisfaction. Issues such as equity of pay or salary levels, promotion practices, hiring, awards, sabbaticals, and institutional or departmental governance are all typical examples of where one may feel a sense of injustice in the workplace (Hagedorn, 2000). Depending on the severity and quantity of these issues, justice can have a dramatic effect on whether a faculty member feels the institution they work for is worth staying at or not. "A sudden realization of inequity serves as a strong 
trigger and likely introduces a strong reaction followed by a significant move on the satisfaction continuum" (Hagedorn, 2000, p. 12)

Change in mood or emotional state. Finally, changes in mood or emotional state are strong triggers that can impact morale and job satisfaction. While there may be little the institution can do to alter mood or disposition, there are strong relationships between one's satisfaction with their job and their current mood. "Thus, mood is a pivotal variable that is strongly responsible for one's location on the job satisfaction continuum" (Hagedorn, 2000, p. 12) Other research has supported this by showing there is a stark contrast between personal happiness and job satisfaction. Most faculty will report low levels of institutional satisfaction even if they enjoy their job (Johnsrud \& Rosser, 2002).

Summary. While many triggers pertain to changes in an individual's personal life, it is still important to note how those changes and perceptions can impact one's perception of their work environment. There is a multitude of ways that institutions can choose to respond to or support the changes that their faculty will experience.

\section{Job Satisfaction Continuum}

Finally, Hagedorn's (2000) framework also indicates that job satisfaction is not absolute, but lies within a continuum. The lowest end of the continuum is categorized as disengagement, the middle categorized as acceptance or tolerance, and the top of the continuum categorized as appreciation of one's job and actively engaged in work. This continuum is fluid and one that is impacted by the mediators and triggers that faculty face throughout their career (Hagedorn, 2000). 


\section{Biomedical Research and Academic Medicine}

Faculty members in biomedical research and academic medicine faculty at academic health centers are evaluated differently and have unique workloads compared to traditional faculty roles (Nyquist et al., 2000). Academic health centers - defined as a center that "encompasses all the health-related components of universities, including their health professions schools, patient care operations, and research" (AAHC, 2014, para. 10) - typically encompass faculty with $\mathrm{MD}$ and $\mathrm{PhD}$ degrees who treat patients, perform basic research, and teach graduate-level students to become physicians or scientists.

While teaching is an expectation of their job, biomedical research faculty at academic health centers are primarily evaluated on the research they conduct, the grants they are awarded, and any patents they might generate. These faculty must also procure their own funding often though federal grants to pay for a portion of their salaries, supplies for their lab, and the salaries of their associated staff (e.g., laboratory technicians, post-docs, graduate students, etc.). Education-related activities often involve teaching courses or individual lectures, mentoring PhD students, and often times developing curriculum for their associated graduate program(s). Many biomedical research faculty choose to not engage in education activities at all, which is often overlooked if they have a strong research or grant portfolio. Changing financial models and shifting federal funding sources have impacted this population's job satisfaction as well as in some cases their mental and emotional well-being (Cole et al., 2009; Holleman et al., 2014; Marburger, 2015; Nyquist et al., 2000).

\section{Funding Mechanisms}

Although faculty typically rate personal compensation fairly low in terms of 
impacting morale (Bender \& Heywood, 2006; Johnsrud \& Rosser, 2002; Rice \& Austin, 1988), funding that impacts their ability to conduct research and scholarship has a much greater impact on job satisfaction and morale (Holleman et al., 2014; Johnsrud \& Rosser, 2002). Funding for biomedical research has seen drastic reductions over the last decade and has significantly impacted how faculty are able to conduct scholarship and how they regard their institutions (Mitchell \& Leachman, 2015).

The various mechanisms in which faculty receive outside funding for their research is often referred to as "soft money." Conversely, "hard money" is money that is appropriated from the institution itself (Stein \& Candler, 2007).

Faculty members in the arts and sciences, business, and law are typically on hard money lines; that is, all or most of their salaries are covered by their school and, in return, they are expected to engage fully in teaching, research, and service throughout their careers, perhaps supplementing their income with grants or awards to cover teaching or scholarly work over the summer. In sharp contrast, many faculty, especially in medicine, public health, and other health science areas, are expected to generate a substantial part of their income through externally funded research grants, or, if they are clinicians, through clinical practice or a combination of practice and research grants. Much or all of these faculty members' incomes depend on the vagaries of federal or philanthropic funding priorities that often may have nothing to do with the priorities of their home institution. (Stein \& Candler, 2007, para. 3)

While some disciplines can expect their work to be paid entirely via hard money, faculty in biomedical research experience a wide array of funding combinations that 
usually involves a combination of the two. For faculty funded mostly or entirely on soft money, there is a persistent need to continually seek out additional funding knowing that the funding one does have will reach a finite point of termination. For those who are exclusively scientists without professional healthcare provider credentials (e.g. physicians, dentists, or pharmacists), there are no alternative routes to generate more funding. The mix of hard and soft money funding can also continue into tenure. Many biomedical research faculty will comment that tenure does not mean much to them as it does not provide the same hard money salary guarantees that colleagues in other disciplines typically receive. Without soft money, one can often expect to lose their position and employment with their institution (Holleman \& Gritz, 2013; Stein \& Candler, 2007).

Most faculty members who conduct basic science research fund their research through federal agencies that grant research dollars. In biomedical research, the National Institutes of Health and the National Science Foundation fund the large majority of biomedical research in the United States (Cole et al., 2009; Holleman et al., 2014; Marburger, 2015). As the primary funding source for biomedical research in the United States, this creates a scenario where many faculty do not receive funding for research and either have to downsize their labs or leave academia. This has created what is called the “death spiral" phenomenon of ever decreased funding (Holleman \& Gritz, 2013, p.614). Essentially, faculty members are forced to gradually shrink their research capacity until none is left.

With decreased availability of federal funding for biomedical research comes increased competition for fewer resources. This means faculty members have to spend 
more time preparing grant proposals and less time doing actual research (Marburger, 2015). This poses an especially difficult challenge for new faculty without established research careers. If one does not already have research funding, they are less likely to be funded in the future. "Every recent study that included how faculty spend their time indicated that faculty perceive they now have less time for both research and teaching" (Nyquist et al., 2000, p. 40).

Many faculty members have also become fearful of potentially losing their jobs or having their programs eliminated. For those who maintain their jobs, it has often times lead to higher course loads, more committee work, additional administrative tasks, and a multitude of other responsibilities. These responsibilities are often reported as having little associated support, act as a distraction from their core teaching and research missions, and are given no credit towards promotion and tenure. The overall feeling of burnout can lead from less productive faculty to those who choose to leave academia all together (Johnsrud \& Rosser, 2002).

While federal policies that dictate how much funds an agency receives are not made with faculty morale in mind, they do come from a combination of other values that must be taken into account. The current federal funding scenario can be classified as redistributive, which is described as taking resources from one group and allocating them to another (Lowi, Ginsberg, Shepsle, \& Ansolabehere, 2013). While having less federal dollars due to less tax revenue is partially to blame, so is a congress that has largely redistributed funds to other priorities such as national defense. John Marburger III, former Science Advisor to President George W. Bush, described that entities such as Homeland Security often have much more political sway with Congress than agencies 
that fund science and education (Marburger, 2015). In times of especially limited federal funding, priority is redistributed to other agencies. While removing funding from the National Institutes of Health was not done with the expressed intent of decreasing scientific discovery and faculty morale in the United States, it is indeed a real consequence (Marburger, 2015).

In summary, federal funding is the foundation in which many biomedical faculty rely upon to complete their work. Without it, a multitude of issues can occur with entire careers possibly in the balance. Understanding how funding has impacted an institution's faculty and in what ways will be important in choosing appropriate ways to respond.

\section{Faculty Wellness}

The stress and anxiety associated with the challenges posed to biomedical research and academic medicine has created scenarios ranging from faculty feeling deflated to departments monitoring each other for suicidal ideology or research misconduct to game the funding system (Holleman et al., 2014).

At the same time the increasing pressures to be financially productive while teaching - or to be funded for research in an increasing competitive environment - have exacerbated stress. And research scientists, who play such an important role in our academic health centers, are particularly stressed by the increased competition for diminishing federal research dollars. (Cole, Goodrich, \& Gritz, 2009, p. viii)

To combat this, many departments and universities have implemented faculty wellness programs of varying scope. At the university level, some institutions have started formal faculty wellness programs that range in offerings from appointments with 
nutritionists to free formal counseling. At the department level, many Department Chairs have begun creating programming for faculty both education and social in scope.

"Several are outside the realm of academic medicine: social gatherings, dance lessons, parenting support groups, opera performances, a faculty art show, meditation, yoga and t'ai chi" (W. Holleman \& Gritz, 2013, p. 614) etc. Ultimately these programs are aimed at mitigating stress, but they can come with an overall cost savings for the institution as well.

An academic medical center's lack of attention to human resources is not only short- sighted, it is expensive as well. The costs of faculty turnover are estimated to be $5 \%$ of a center's budget, not including the costs of lost opportunities, lost referrals, overload on remaining faculty, reduced productivity, and lower morale. (Cole et al., 2009, p. 5)

With human health at the core of academic medicine's mission, faculty wellness programs can play a role in how biomedical research faculty report job satisfaction and morale. Programming can be offered in a multitude of ways, but finding what programming is most impactful and needed requires understanding how an institution's faculty describe their own morale and job satisfaction.

\section{Academic Health Center Priorities}

Conducting biomedical research at an academic health center is a double-edged sword for faculty. On the one hand, it is the perfect place to conduct research on human health as novel findings in the lab could potentially be brought to clinical trial in-house and eventually lead to a new therapy to combat human disease. Scientists can work with any number of healthcare practitioners to better understand a condition and collaborate in 
clinical settings not available at a traditional university environment (Nyquist et al., 2000). On the other, research faculty must operate in an environment that often times prioritizes the clinical enterprise much higher than that of basic research. Academic health centers typically have three primary missions (education, patient care, and research), which when one mission becomes a higher priority than others (typically patient care) can lead to research faculty feeling like second-class citizens at their own institutions (Cole et al., 2009; Holleman et al., 2014; Nyquist et al., 2000)

When resources are scarce, biomedical research faculty are often the first to have funding removed or be pressured to apply for more grants. Since a clinician can see more patients to generate more revenue, academic health centers will prioritize that first and foremost. Combined with the national push to reform healthcare delivery and how care can be reimbursed, the needs of researchers at academic health centers are often ignored or left to individuals to resolve on their own (Cole et al., 2009; Holleman et al., 2014; Marburger, 2015). With educating graduate students (which includes the expectation to fund them) often a secondary priority to conducting research, teaching and mentoring diminishes as well (Smolka, Halushka, \& Garrett-Mayer, 2015).

\section{Summary}

While the predominant funding mechanism for biomedical research is indeed an issue, it is not the whole picture in biomedical research job satisfaction. The priorities of academic health centers as well as how institutions respond to faculty wellness issues are equally important. Ideally an academic health center is addressing all three, which is important when taking into consideration perceived faculty morale and job satisfaction issues. 


\section{Conclusion}

This chapter reviewed the foundations of morale and job satisfaction research, a conceptual framework specifically designed to review morale and job satisfaction of university faculty and the corresponding literature, as well as factors that impact biomedical research faculty at academic health centers. While much research exists on faculty job satisfaction holistically, little research has been done specifically on what contributes towards high morale and job satisfaction for faculty members in biomedical research programs. This approach is consistent with recent research focused on researching morale and job satisfaction of faculty who work in specific disciplines and institution types. With competitiveness for funding and concerns for wellbeing at reportedly high levels, knowing more about what influences satisfaction and morale for this population of faculty is critical in maintaining healthy, happy, and productive employees. This study addresses that problem by contributing new research on what may uniquely impact this population of faculty and what academic health centers may be able to do to improve their own environments. 


\section{CHAPTER 3: Methodology}

While there is a solid foundation of knowledge about what influences morale and job satisfaction for faculty who teach at traditional undergraduate colleges, only recently have issues pertaining specifically to faculty members in biomedical research fields come to light. It is unknown whether general faculty job satisfaction best practices equally suit biomedical research faculty members or whether there are unique factors that contribute to higher morale and job satisfaction. This study is also the first study to the researcher's knowledge to specifically assess job satisfaction and morale of biomedical research faculty at an academic health center. This chapter describes the study's research questions, its methodology, how participants will be selected, how data will be analyzed, and research bias.

The purpose of this study is to explore the personal and professional factors that contribute to positive morale and job satisfaction for faculty in biomedical research programs at academic health centers. The two research questions this study addresses are:

1. How do biomedical research faculty at an academic health center describe the mediators and triggers that influence their morale and job satisfaction?

2. What are the differences in morale and job satisfaction of biomedical research faculty by gender and length of employment at their current academic health center?

These research questions address gaps that exist in current literature and through answering the questions it is possible to make recommendations as to how academic health centers can increase job satisfaction and morale (Holleman et al., 2014; Nyquist et 
al., 2000). The first question compares responses from biomedical research faculty on their self-identified mediators and triggers as described in this study's conceptual framework (Hagedorn, 2000). The second explores responses by length of employment and by gender to see if differences exist between biomedical research faculty (Bender \& Heywood, 2006; Callister, 2006; Fox \& Colatrella, 2006; Ropers-Huilman, 2000; Sabharwal \& Elizabeth, 2009).

This was a qualitative study utilizing semi-structured, in-person interviews with biomedical research faculty. A qualitative approach is most appropriate for answering the study's research questions as it allows for the exploration of values and feelings that influence perceptions through full narratives and stories (Glesne, 2015). Qualitative research has also shown to be effective in conducing discipline-specific faculty research for faculty in a limited geographic area (Shockness, 2015).

A quantitative approach was selected due to the scarcity of evidence needed to appropriately inform outcomes measures such as survey items. Furthermore, faculty would be constrained due to the nature of standardized quantitative job satisfaction surveys. Most existing studies on morale and job satisfaction for faculty in STEM fields were quantitative and use variations of popular established surveys (Babin Verret, 2012; Bateh, 2013; Brannen et al., 2014; Foster \& Lyman, 2012; Johnson, 2010). While some instruments offer the option for open-ended responses; that too limits the opportunity to ask for further clarification or additional information. The environment of an academic health center is significantly different from traditional comprehensive universities and liberal arts colleges, thus existing quantitative tools such as surveys may fail to capture the nuanced differences between the various environments. 
Finally, the site where this study was conducted had recently released the results of a faculty job satisfaction survey conducted approximately one year prior to this study. The results of that survey were described by faculty as misleading or sometimes fabricated, which could lead to potential distrust of future surveys or survey fatigue. Allowing faculty to describe aspects of job satisfaction themselves without the limitations of Likert-type scales leads to more detailed and descriptive responses. Comments regarding this survey appear multiple times in the results section.

\section{Methods}

This qualitative study was done through a series of interviews exploring faculty relationships with their professional environments using Hagedorn's (2000) Conceptual Framework for Faculty Job Satisfaction. Using its established framework of mediators and triggers, responses from faculty were categorized and analyzed using existing analysis methods. Identifying which responses overlap and which differ paints a clearer picture of how faculty perceive their own job satisfaction and morale by rank and gender. This section will describe how participants were selected, the interview protocols used, how data was stored, and how interview data was then analyzed.

\section{Participant Selection}

The population for this study was tenure-track graduate faculty in biomedical research programs whose faculty appointments were primarily research-based. Participants included reflect all three tenure-track ranks and are either current or former principal investigators on research grants. Excluded in this study were faculty with dual clinical appointments (those who see patients as well as conduct research), professional clinical licensure (physicians, dentists, nurses, pharmacists, etc.), or administrative 
appointments that provide hard money FTE support (Program Directors, Department

Chairs, etc.). Due to the relatively rare nature of appropriately credentialed individuals in the general population as well as a limited number of academic health centers, 12 biomedical research faculty were purposefully selected at one academic health center in the Western United States. Due to the researcher's professional role, purposeful selection in this study was chosen to build on established relationships with eligible participants, which led to productive results and better answer this study's research questions. These rationale satisfy commonly used criteria for choosing purposeful over random sampling for qualitative research (Maxwell, 2013).

Due to inconsistencies in how tenure and promotion is managed at this institution, faculty results have been divided not by rank or tenure status, but by length of employment. Six male and six female faculty of varying lengths of employment were interviewed to analyze differences in responses by gender and length of employment. It is important that length of employment at current institution and gender are as evenly distributed as possible, which would not have been possible via random sampling.

\section{Interview Protocol and Data Collection}

From the sample population, faculty were purposefully selected and were either emailed or asked in person whether they would like to participate. Twelve faculty accepted and all participants received an official email invitation for their records (see Appendix A) as well as an informed consent document (see Appendix B) that they signed before participating.

Each participant chose a time and place for their interview. Each interview started with a review of the study and signing of the consent form. The audio recording device 
was then turned on and I introduced myself followed by reiterating the purpose of the study. Questions covered a variety of topics related to Hagedorn's (2000) mediators and triggers. Audio recordings were then transcribed verbatim for analysis.

Table 1 describes each participating faculty member's gender, length of employment at their current institution, as well as an assigned pseudonym. Six male and six female faculty accepted to participate as well as equal distributions of 5-year current employment intervals $(0-5,5-10,10-15$, and $15+$ years $)$.

Table 1

Participating Faculty by Gender and Length of Employment

\begin{tabular}{lll}
\hline Name & Gender & Length of Employment at Current Institution \\
\hline Dr. Grey & Female & $15+$ years \\
Dr. Thomas & Male & $10-15$ years \\
Dr. Martin & Female & $10-15$ years \\
Dr. Anderson & Female & $5-10$ years \\
Dr. Parker & Male & $0-5$ years \\
Dr. Black & Female & $0-5$ years \\
Dr. Davis & Male & $10-15$ years \\
Dr. Johnson & Male & $15+$ years \\
Dr. Banner & Male & $0-5$ years \\
Dr. Smith & Female & $5-10$ years \\
Dr. White & Female & $5-10$ years \\
Dr. Miller & Male & $15+$ years \\
\hline
\end{tabular}


The questions used for this study (see Appendix C) were designed to answer this study's research questions using Hagedorn's (2000) framework of mediators and triggers. Each question was open-ended to elicit information about the participant's experience. Follow up questions or questions to further explore a response were also used to gain indepth and rich data (Glesne, 2015). The questions were arranged in an order intended to make the conversation naturally flow while also balancing questions that may speak to more personally sensitive issues.

The following questions (1 and 6) were designed to elicit responses related to triggers - specifically issues pertaining to change. While all questions asked pertain to this study's first research question by having participants describe triggers that influence their morale and job satisfaction, question 6 had the potential to elaborate on this study's second research question about differences between gender and ethnicity.

1. Think back to when you first discovered your interest in biomedical research. What initially interested you in this career and what motivates you now?

6. Describe ways in which you feel your career choice has impacted your personal or family life.

The following questions $(3,4$, and 9) were designed to elicit response related to motivators and hygienes. Specifically these questions touch on issues of how they describe their work as well as how they are recognized for the work they do. Question 9 was intentionally designed as the last question as it should generally result in a positive response and end the interview with the participant potentially feeling more positive about the experience. Ending on a potentially negative note could decrease a 
participant's willingness to answer follow up questions at a later time or diminish rapport with the researcher (Glesne, 2015).

3. Describe to me how you prioritize your work

4. Tell me about how you are recognized for your work.

9. What is the most rewarding aspect or accomplishment you have made related to your work as a scientist?

Question 5 was the only question asked specifically related to demographics. While demographic information was already obtained prior to the interview and can certainly appear as a theme in other questions, asking directly how they feel their gender or ethnicity has played a role in their work environment helped better answer this study's second research question.

5. Can you identify instances in which you feel professionally you have been treated differently due to your gender?

Finally, questions 2, 7, and 8 were about environmental conditions. They also are related to relationships that faculty have in their work environment. These questions asked about relationships with administration, their colleagues, and their overall feeling about the campus environment.

2. Describe what you find appealing about working at an academic health center as a scientist compared to other environments.

7. Leadership and politics typically change over time at colleges and universities. Tell me about your relationship or perspective historically with the university's administration.

8. Similarly, tell me about the kind of relationships you have working with other 
faculty.

Overall, these questions provided opportunities for participants to elicit responses related to both of this study's research questions as well as Hagedorn's (2000) mediators and triggers. The semi-structured nature of these interviews allowed for individualized prompting for more information if any question results in short answers or is misunderstood.

Audio recordings of the interviews were captured on an electronic recording device and transferred to Portland State University's secured Google Drive cloud service. All audio recordings, transcriptions, and other research-related documentation are stored on Google Drive and will be destroyed one year after publication of the dissertation.

\section{Data Analysis}

Analysis of interview data was done through a combination of initial, values, and magnitude coding. Audio recordings of interviews were transcribed and coded to analyze trends and themes that showed strong relationships to increasing or decreasing job satisfaction and morale. Coding of transcripts was conducted using ATLAS.ti 8 qualitative data analysis software. Responses were categorized (using initial coding) according to Hagedorn's (2000) mediators and triggers (using values and magnitude coding) to determine which types of responses indicated the highest increases in morale and job satisfaction and which indicate higher dissatisfaction.

Initial coding. Initial coding (Saldaña, 2016) was then used to categorize responses into codes most resembling Hagedorn's 20 pre-established mediators and triggers (Hagedorn, 2000) (see Appendix D for examples of coding). Responses were given more than one code or no codes if appropriate. If enough responses did not fit into 
Hagedorn-associated codes, new codes were established in order to analyze these new categories of responses. Subsequent coding methods were then done sequentially.

Values coding. Value coding (Saldaña, 2016) was then be applied to responses in order to gauge a participant's attitudes, values, or beliefs about the question they were asked. Value coding was also used to gauge whether participants felt certain questions pertained to personal values, professional values, or otherwise (see Appendix D for examples of coding). Assessing values associated with responses is especially important in understanding morale and job satisfaction as categories with the highest perceived value will likely have more influence over morale and job satisfaction than others. Most importantly, values coding helped prioritize what to improve on and what can wait or not be addressed.

Magnitude coding. Finally, magnitude coding (Saldaña, 2016) was used to assess whether a response indicated a positive or negative relationship with its associated mediator or trigger. Magnitude coding was used as it is often used for "sentiment analysis or opinion mining, which examines positive, negative, and neutral perspectives" (Saldaña, 2016, p. 86). The specific codes used are: POS - Positive, NEU - Neutral, NEG - Negative, and MIX - Mixed. Like values coding, magnitude coding also helps prioritize what issues related to job satisfaction to improve on and what can wait or not be addressed (see Appendix D for examples of coding).

Data from interview transcripts was sorted by Hagedorn's mediators and triggers, implied expressed values, and then assessed magnitude codes. The majority of responses fell within the initial coding criteria with only a few unrelated themes falling outside. Responses within each mediator and trigger were then sorted by gender and then length 
of employment at this institution to identify differences in responses.

\section{Limitations}

This study was limited to faculty who held tenure-track faculty appointments and were willing to volunteer their feedback regarding morale and job satisfaction at one academic health center. This study also excluded any faculty with dual clinical appointments, which further limited the number of qualified faculty to interview. Because of the sample size, it was not possible to address issues regarding differences in morale and job satisfaction by race or ethnicity. Finally, due to the nature of how one may perceive repercussions or potential retaliation for speaking negatively about their employer, there may have been concerns not expressed due to perceived negative consequences.

This study was conducted with faculty at one academic health center located in the Western United States. Results may not adequately capture issues relevant at all academic health centers due to campus type, geographic location, local culture, and issues specific to that institution. It may also not capture issues that are important to biomedical research faculty with dual clinical appointments or faculty who also hold dual administrative appointments that supplement their funding. The results from this study may be transferable to other academic health centers and the study protocol can be replicated at other academic health centers.

\section{Researcher Bias}

As with any qualitative research study, it is important to identify one's own bias and disclose up front any conflicts of interest (Maxwell, 2013). It has also been said that to separate one's research from other aspects of their life can "cut you off from a major 
source of insights, hypotheses, and validity checks" (Maxwell, 2013 p. 45). As a student in Portland State University's Doctor of Education program, we were encouraged to explore research topics that represent issues within our own roles as professionals. Mixing roles as a student and a professional exploring one's own professional interests obviously brings forth bias, but also those insights, hypotheses, and validity checks that add their own strengths to this type of study.

In the case of this study, working with faculty that seem unhappy or frustrated professionally is something that I feel has been a significant issue in my professional role. Helping faculty and working along side them has proven to be a source of great personal satisfaction and something I feel I am particularly good at in my professional work. It is a core function of my job to be aware of any issues that can negatively impact our learning environment, so being aware of what might be detrimental to the experience of our faculty is something I can potentially use to improve the learning environment.

I also bring with me a history of working with graduate faculty in biomedical research programs and my own ideas of what kinds of answers to expect. I have a personal interest and fascination with scientific research and have dedicated my professional life to helping it move forward, so I do have a vested interest in finding answers to contribute to a positive scientific research environment. That said, I expected to suspend these biases by objectively coding the responses I received to look for shared relationships and not focusing just on responses that reflect my expectations (Saldaña, 2016).

What I also needed to disclose up front is my role in these interviews as an impartial doctoral student and not necessarily a representative of a university or 
somebody in a position of perceived power. I felt that I already had positive rapport within the community of faculty who may be participating in this study, but they needed to know that they can trust me to keep their responses completely confidential. Not having that trust or being perceived as somebody who has to report results to higher administrators could have led to censored responses or lack of adequate participation.

\section{Conclusion}

The purpose of this study is to explore the personal and professional factors that contribute to positive morale and job satisfaction for faculty in biomedical research programs at academic health centers. This study explored those factors by using semistructured interviews with 12 faculty. Those interviews were then transcribed and coded using open, initial, values, and magnitude coding methods. Coded data was then used to answer this study's research questions and explored how personal and professional factors relate to positive job satisfaction and morale. 


\section{CHAPTER 4: Themes of Job Satisfaction}

This chapter presents the data from the 12 faculty participant interviews. While the participant responses did not always agree with all the components of Hagedorn's (2000) conceptual framework - it is a useful structure for presenting the data because what did not align is equally important to measure faculty job satisfaction. Responses aligned closely to Hagedorn's (2000) mediators, but not as strongly to the framework's designated triggers. Data presented in this chapter is organized by Hagedorn's (2000) framework to answer the research question about how mediators and triggers influence faculty morale (research question \#1) and differences in morale of faculty by gender and length of employment (research question \#2).

\section{Mediators}

During our interviews, faculty expressed much of their satisfaction and dissatisfaction related to issues or situations that impact interactions with colleagues or their environment. As such, mediators (variables that produce an interaction effect) play an important role in assessing job satisfaction for this population. Responses were sorted into motivators and hygienes, demographics, and environmental conditions as outlined by Hagedorn's (2000) conceptual framework.

\section{Motivators and Hygienes}

Motivators and hygienes (demotivators) are mediators, meaning they are variables that influence relationships through interaction effects. Therefore these variables are used to describe types of interactions that faculty have with either their colleagues or their environment (e.g. their employer, funding agencies, or otherwise). Achievement, recognition, work itself, responsibility, advancement, and salary were all coded for and 
assessed both values and magnitude regarding job satisfaction. Faculty interviewed expressed high value in recognition, achievement, work itself, and responsibility. Advancement and salary, while mentioned, held less value and only marginally influenced job satisfaction.

Recognition. None of the faculty interviewed in this study felt that they were adequately recognized for their work or achievements. Recognition was sorted into internal recognition from their institution and external recognition from entities such as professional organizations or colleagues at other institutions. Internal recognition was described as lacking and was therefore coded as a demotivator. Many could not name an instance where they were internally recognized, while others only mentioned being thanked for doing regular tasks or receiving teaching wards. Consistent with others, Dr. Grey expressed the lack of internal recognition.

I'm actually just trying to think about instances where [the work I do] is recognized other than people saying, "thanks for taking care of that." I'm completely serious. Like, someone will say "thanks for sending that email out for me" but that's sort of all of it. There's an expectation that you'll do things and maybe if you didn't do them then you'd hear about it. Then it's more of a punishment contingency than a positive reinforcement contingence. (Dr. Grey) Similarly, some faculty felt that some internal recognition can be a demotivator-being good at your job can lead to additional committee assignments or administrative tasks. Not all faculty want to be distracted from their research by other activities. Institutionally I'm not sure that I'm necessarily recognized, but apparently my name has floated to the top of lists of people to bug when you need someone on a 
[institutional] committee. (Dr. Anderson)

For the most part, recognition for work was described as almost entirely external for the scientific work they publish. Most often this recognition was described in the form of publications, grants, awards, or invitations to speak on their topic of research. External recognition is certainly a motivator and expressed as a positive value by all faculty interviewed.

For me what's most important is that first publications come out of a lab will be cited so people will start to understand what type of work it is we do and that is meaningful. That then sort of trickles into whether people see you as someone they want to start to invite to speak at conferences. That's something I really enjoy doing - is talking at conferences and I have started to get invitations based on the fact that people know I'm one of the few people doing my very specific thing and I think that's a really important part of the recognition. (Dr. Banner) Other faculty also shared that external recognition for the science one is conducting can be the ultimate goal for faculty.

I mean grants you have to get, but it doesn't really seem to matter who you are or which institution you work at. I think its really when people start to see your science and start to recognize you as potentially the person to go to if they have questions about whatever it is you do, that's really the pinnacle. That's what you're trying to get to and how your field recognizes you. (Dr. Parker) A frustration shared with many faculty is that although it is easier to identify instances of external recognition, the lack of internal recognition can be demotivating. One professor specifically cited their frustration with no internal mechanism that supports 
departmental momentum or collective achievement.

If I got a grant this morning, how does my department benefit? Is there any money that's going to come to the department for that success? No. Zero. What about my group? Can I say because of my group's collective success we can have a happy hour? Nope. Not one thing. There is zero reward. That is not the same at other places. Having that type of reward system built in for when people do rise up collectively, that's important for social dynamics. (Dr. Davis)

Together it appears that recognition both internally and externally are highly valued and can positively add to job satisfaction. Conversely, lack of recognition can be demotivating; especially in workgroups where they feel their collective success is not recognized or rewarded by the university. Overall, faculty have a much higher sense of external recognition through publications, grants, and invitations to speak than any form of internal recognition.

Achievement and work itself. Faculty unanimously described that the work itself and the achievements associated with it were what gave them the greatest sense of job satisfaction. The work itself was enjoyable, often regarded as fun, and deeply enriching. Many participants shared that the work they do provides opportunities to work in different settings such as private industry, but conducting science at an academic health center is specifically what they find most rewarding. One common sentiment was that the work itself outweighs any of the tangential negative aspects.

I'm absolutely still motivated by conducting scientific research because if it weren't fun and really enjoyable, it wouldn't be worth some of the crap. And I can't be the first person who has said that. When it stops being fun I stop doing 
this. I can do other things in my life. (Dr. Anderson)

Other faculty expressed similar sentiments, but also extended the notion that the excitement holds true for both current and past discoveries. It was also expressed that many faculty share the excitement with their colleagues and that energy helps motivate each other towards the next discovery.

So, scientific discoveries, some of the best stuff we've done we're doing right now because it's finally translating into something. So, for me, right now when everything seems to be kind of down in terms of funding and the environment, that's what keeps me going thinking, "this is some of the coolest stuff we've ever done." It's really exciting and you want to tell everyone about it. There's also some stuff we did early on that I'm still really proud of and like to go back and look at and say, "I did that!" (Dr. Martin)

Some faculty described not just science as a motivation, but the associated education mission working at an academic health center. Many faculty volunteer their time doing scientific outreach activities or hosting students during Summer research internships.

I'm still motivated about the science, so when I do outreach with the kids I remind them that's one of the only jobs where you get paid to mix a bunch of things together to see what happens. It's kind of fun and exciting. And you get paid to find out what nobody else knows about and that still drives me. I think what drives me to do this in an academic setting is my passion for education. You know, we've all looked at other opportunities in pharma or private foundations and you always miss that interaction with students and kind of the education 
culture, which I think is so important and an important way for scientists to kind of give back. (Dr. Black)

Also associated with conducting science at an academic health center is the sense of meaning that comes from what one's research can lead to. All faculty responded with a sense of meaning and pride that comes from research that contributes towards the larger goal of ultimately helping people.

What also helps me is I really like having a larger goal to help people. Sometimes in your day-to-day work it's very frustrating, but if you have that larger goal that the knowledge that you're gaining or the material that you're working on could someday help people, that kind of sustains me and my research. (Dr. White) Ultimately, all faculty reported being driven by the work itself. Scientific research is exciting, it provides opportunities to train students and future scientists, and biomedical research provides a way to contribute towards the larger goal of helping people. The work itself — conducting science - is a strong motivator.

Responsibility. Autonomy is a highly valued job aspect that all faculty described as an element that provides satisfaction and motivation. While all faculty directly report to a Department Chair or Director of some sort, they all expressed great satisfaction from the autonomy that comes with running your own laboratory the way you want.

Autonomy is what initially motivated them to pursue academic research in the first place. Probably part of what interested me in biomedical research was the independence of thought. Like I knew if I was a scientist I thought I'd get to choose what I work on. You know it was kind of control over what you found interesting and you would kind of go down that path - the thrill of discovery. So scientifically I 
have full autonomy and it's great. (Dr. Black)

Faculty often described the autonomy over one's work environment to be highly valuable when raising children. Being able to come and go without having to be present for set hours was often mentioned.

So part of the reason for staying in academics is there is a lot of scheduling flexibility. Which, so when we moved here I had [two small children] and we were fairly fortunate to get pretty good childcare. It was before [this institution] had childcare. So we found a nice one, but when they got to half-day kindergarten I was having to leave in the middle of the day every day to go transport them to a different daycare. In a normal 9-5 job you wouldn't be able to do that. And the same with when they were in middle school and playing soccer and had to leave at 3 to get to the soccer practice or game or whatever. So you have that flexibility. (Dr. Martin)

However, with autonomy also comes a sense of never ending work. Many faculty described flexible hours positively, but noted that with that flexibility also comes a sense of being available at any time to work. An often-described byproduct of that is their children express disinterest in pursuing scientific research as a future career.

In some ways academia is great. Having children, the flexibility is phenomenal, so I really appreciate that part of the job. It has enabled me to see my children more and to do more with them. My daughter hates my career and never wants to do it. It never ends. There's always something there. Overall the flexibility has been great and far easier for me than another industry and I'm very appreciative of that. (Dr. Smith) 
Overall, the responsibility that comes with autonomy to conduct one's research in the way they want is a highly valued trait of the work environment. Autonomy also allows scheduling flexibility valued by faculty raising families. Autonomy is a strong motivator that contributes positively towards the work environment and is described as a one of the most appealing aspects of biomedical research at an academic health center.

Advancement. Advancement itself was not expressed as an issue of high value nor as a motivator of morale. While some faculty expressed that they would like to have tenure, most acknowledged that tenure does not make a difference for biomedical research faculty due to the nature of their grant funding. Tenure for biomedical research faculty at academic health centers often does not include any difference in hard money or guaranteed salary protection, so tenure itself is not highly valued nor is it a motivator. In addition, it is not necessary to get tenure to stay in their roles. Faculty who have been at this institution the longest all talked about advancement largely in terms of one's ability to maintain grants and little to do with contributions to the education mission.

When I came here I was so naive about it, but I think it's become a lot more difficult to get grants. I think everybody is or most people are kind of scrambling and I think that your value as a faculty person is really weighed in terms of how much money you are bringing it. So it doesn't matter you could be educator person doing wonderful service or have tenure, but I think that if you're not bringing in grants you're not going to have a job. I definitely didn't feel the sort of pressure earlier in my career that I feel now. (Dr. Grey) In summary, biomedical research faculty at this institution do not highly value tenure or advancement in rank due to its low impact on their worklife or ability to 
continue in their positions. The pursuit of grant or other external funding is much more important to secure stable employment. Retrospectively, faculty view their earlier sense of naivety about the bigger picture of how difficult it can be to not just obtain, but to sustain funding over the duration of one's career.

Salary. Salary itself or the amount faculty are paid was not expressed as a common concern nor a motivator. Almost all faculty expressed sentiments stating that they knew going into academia would not be their most financially lucrative option and were comfortable with their choice. Desires for larger salaries were jokingly stated or welcomed if offered, but salary itself did not present itself as an element that influenced job satisfaction.

The much larger and universally acknowledged aspect of salary is not the salary itself, but how salary is calculated, how much comes from university, and stability in salary proportion. All faculty interviewed received only a portion of their salary from the university through hard money and the rest came from their own grant funds (i.e., soft money). Many expressed frustrations with this arrangement not just because of the split in salary funds itself, but how that split between hard and soft money is administered. Faculty from this institution had recently negotiated a new salary contract that increased institutional hard money up to $50 \%$ of their salary or more, so the new contract was frequently cited.

I've paid part of my salary ever since I started here because I came with a type of award where the expectation was that I was going to pay part of my salary off of that. So I have ranged from $75 \%$, covering $75 \%$ of my salary off of grants to now $50 \%$, and that's a huge thing. That's basically so I can afford at least half a 
technician sort of thing. So I think that's really great and having that in writing and knowing it's going to be $50 \%$, that's, I think to be honest there was a lot of inconsistency with how different basic science departments in terms of how much is being covered and the faculty salary equity was a little like, this, and I don't know if that completely solved that situation. (Dr. Anderson)

While some faculty like Dr. Anderson saw the new contract in a largely positive light, others felt like it was not enough of a commitment. Many faculty expressed opinions similar to Dr. Davis.

I get cobbled together about $50 \%$ of my support is coming from [the institution]. About $50 \%$ of my time is covered. I've been here [many] years and I'm not tenured. I'm on a yearly contract, which is fine. There's no financial investment or commitment from the university level to me. (Dr. Davis)

The other frequently cited source of salary frustration was that teaching at this institution was not paid. Although an expectation included with the hard money funds they receive from the institution is to contribute to the academic mission, this is rarely enforced nor does it seem to matter for annual reviews or promotion and tenure. While not getting paid additionally to teach is cited as not surprising or expected by most, they see colleagues in professional health programs at the same institution who receive additional funds for teaching on top of their clinical work. This led many to express beliefs of unfair treatment or a sense of teaching basic science courses to be of low value to the institution.

Nobody ever gets paid to teach, which to me is very high-level job expectation and we get no money for it. You wouldn't expect a nurse to be on duty and not 
get paid for it, but you expect me to teach five courses and not get paid for it. That doesn't make any sense. And so they're like, "there's no profit in it; you're already here" kind of thing. So you're charging students tuition and I don't see any of that. (Dr. Black)

Although most faculty feel it is unfair that they are not compensated for teaching in the same way as their healthcare provider colleagues, most express that they genuinely enjoy teaching and find it fulfilling. Teaching itself is described as a motivator, but the inequity in how faculty are compensated is a demotivator. The word "volunteer" was used many times in regards to teaching as it was not perceived to be an activity one was compensated for.

Teaching is part of the reason I'm here. It's one of the ways we advance the field and train young scientists. I like teaching and put a lot of work into it. Most faculty participate in teaching as volunteers because it makes no difference in your salary. Most people do it because they think it's an important part of the mission. There's a certain element of idealism to that. (Dr. Johnson)

Finally, most faculty expressed frustration with policies that impact how their grants are managed. Many shared sentiments about how administrative decisions regarding funding can negatively impact how grants are fundamentally managed. This dissatisfaction was typically expressed with a sense of perceived low value and lack of understanding from the institution for the work they do.

I think the other thing that I've seen happen too many times here is where they put people in place to make policy changes that they have never actually experienced what this policy is going to change. Like you have people who are making 
decisions of how we're getting our salaries paid. So a couple of years ago they were like, "we're going to give everybody merit increases and we're going to get everybody up to speed on national levels of salaries" or whatever. And they say, "oh, by the way, you have to start paying that out of your grants starting next year." Well that's not how grants work. Grants have a five-year budget and you're stuck in that budget. You can't just mid-way through that budget say you're giving yourself this raise to bring me up to national levels and the university says it has to come off this grant I have. The grant organization is going to say, "you're kidding, that's not how it works." So they implemented all this stuff and nobody who is funded by federal grants could do it because they didn't understand how grants work, which is pretty naive. (Dr. Martin) Beyond general principles of grant management, many faculty also expressed general frustrations with how grants are divided and appropriated. Many mentioned examples of splitting grants into single digit percentages and spreading those funds across multiple projects and staff for unknown reasons.

I got an email earlier this week saying you're losing $2 \%$ from [a colleague's] grant, their grant finishes at the end of February, where do you want that $2 \%$ to come from? That sort of thing is going on all the time. And later today I have to write an email saying we got a $10 \%$ budget cut, and you know how you were on the $3.7 \%$ FTE? Well, now you're only going to be on for $1.25 \%$. What the hell am I doing having to do that? (Dr. Grey) In summary, faculty interviewed are largely not motivated by salary itself. They entered the profession knowing it had typically lower wages and accepted that as the 
nature of the context. What does frustrate faculty is how their salaries are determined and which activities contribute towards their salary. Not getting paid equitably for teaching as well as administrative decisions that do not take into account the nuanced nature of long-term grant funding mechanisms negatively impacted job satisfaction.

\section{Demographics}

Unlike other mediators, demographics (especially in regards to gender and ethnicity) are typically constant and stay fixed. This can lead to the potential for discrimination or the creation of in and out groups. How institutions support their faculty of different demographics and the culture of each work environment can lead to drastically different responses in regards to job satisfaction. This institution in particular elicited many responses regarding differences in how female and male faculty perceive job satisfaction as well as comments about the institution type.

Gender. All faculty expressed that male and female faculty experience differences in their work environment. Namely, female faculty felt like they take on a greater teaching load, participate on more committees, and miss out on advancement opportunities due to feeling less comfortable with self-promotion. Male faculty members all expressed that they acknowledge their gender has likely helped them and that their female counterparts experience disadvantages, but they do not feel like they are empowered to change that. "Absolutely. People who tell me [gender] is no longer an issue have no idea what they're talking about. Um, yeah I think women in science are still facing an uphill battle. It's frustrating" (Dr. Martin). Other female faculty expressed similar concerns.

I would say yes, absolutely gender plays a role around here. I have very little 
doubt about that. I think that it probably affects things negatively. I think you get asked to do more things and maybe it's an upbringing thing, but it's hard to say no. I've noticed my male colleagues don't have a problem saying "no" and they also feel...I think that they also don't feel any hesitation for asking for things. When I was on [a committee] you would hear about someone asked for something and you're thinking "I would never think to ask for that" or "I've been doing that for free!" (Dr. Grey)

Other female faculty shared examples of when they feel they were blatantly discriminated against for being a woman. Having their grant submissions reviewed under what was perceived as discriminatory criteria was expressed multiple times. As described in the following example, none of the women faculty interviewed reported these instances to their institution officials or felt like doing so would make a difference.

Oh yeah. Once upon a time I submitted a grant internally here for just a tiny amount of money. One of the reviewers, I don't know what decade they dug him out of, but he said that, "she seems a little young to be running her own lab." That was literally written as the review, written, yes. And I was so pissed off, so pissed off because that's so inappropriate on so many levels and it's in writing. I'd say yes, I've been discriminated. I'd hazard if you talked to any woman on this campus they have had that. We seem to be shedding the cranky old men from the department and the institution, but, you know, there historically have been just dirty old men that don't realize you can't say things like that. I'm not the type of person to go report people, but just little things like, "really?" (Dr. Anderson) While female faculty still express feeling of being disadvantaged, senior faculty 
were often quick to express that things have gotten better over time and that gender differences are diminishing. Female faculty at this institution also often cited examples of having women in leadership roles, which has improved the environment for other female faculty.

I think [gender discrepancy] was definitely biggest when I was younger. I'm more aware of it as a faculty member than I probably was as a graduate student, but I see it less now. Here I have lots of female colleagues and there are many females in leadership roles. There's still this feeling, though, that male leaders will ask other males out for beers or out to golf and you're not involved with that. It's still there a little bit, but not as much when I started. (Dr. White)

Conversely, some faculty acknowledged that some women in leadership roles express the same leadership qualities as their male counterparts. This was expressed as a demotivator more than a motivator.

If you look at a lot of the women in power on this campus, [gender discrepancy] doesn't matter to them. And that's not how they got there. And unfortunately a lot of women in power in science are women who have male attributes. They're very good at self-promotion, which women tend not to be. That's what is rewarded. So we often in some of the women's circles on campus, we talk about if you really want [to advance] you'll have to behave in a certain way because this is what they respect and that's how you get there. And there are some of us that just won't do that. (Dr. Black)

Raising a family is a concern that was addressed by all but one female faculty member interviewed, but only one male faculty. While autonomy has already been 
described as an element that provides flexibility for faculty to raise families, there are still concerns in terms of career advancement and grant attainment.

A big thing I have fought here and I know the Provost was kind of aware of; the Provost was a supporter of fixing scheduling meetings when moms can't come. So, I had this happen to me in the last three months like four times when they want to meet at 7am while I'm trying to get my kids ready to school or the bus. The last one was a workgroup they wanted to start at 6pm. Well, I'm sorry that's when I have dinner with my kids or I have to pick them up. That's been going on continuously. It's worse here than when I was at [a previous institution], which is surprising. Here it's been very unfriendly and that's not something I haven't told everyone. I think that they just don't care. (Dr. Martin)

The one male faculty member who commented on raising a family mentioned how unfair the national grant funding system, not the institution, is to women who might choose to pause their career and start a family. He also shared what in his mind was a disparity in how other women view taking breaks to raise a family compared to men.

We push a lot on our work, so when does [a women who wants to start a family] take this break? Science doesn't care if you take a break or not. You can take a break and still be a great scientist. But money, your competition, you're getting judged on every aspect 24/7. So you're competing against someone who on Saturday night is sitting there writing grants. So what if you want to be at Chuck E Cheese with your kid? How do you? That's nasty. That's the nasty part and that's just Saturday night. If you want to take off maternity leave and these other things, these people are passing you by. The biggest critics I've seen to this aren't 
the men. They've never. It's women. It's that 60-year-old female Harvard professor who says, "I didn't get that, you aren't getting that." There is no mercy on the women-to-women side. No mercy. Because they're like, "I gave up everything and destroyed all of my personal life and you think you want this now? No way." Yeah, that is not a man thing. Man-to-female thing no problem. But women-to-women, no way. (Dr. Thomas)

While female faculty expressed personal instances of feeling disadvantaged as a women, male faculty were largely quick to admit that their gender likely did not disadvantage them. "It's doubtful [being male] made any difference in terms of promotion and tenure. Did it make it easier for me to interact with some members of the past administrations? Yeah, but I don't think it benefitted me much” (Dr. Johnson).

Most male faculty spent more time describing ways in which their female colleagues or students were at a disadvantage and what they as men either make them aware of or what they have observed. No male faculty felt especially empowered to do much about the disadvantages female faculty face other than to set a better example in their own personal work environments.

I have no problem putting my student out in the spotlight whether they're male or female and I know that female students has to perform a little bit better. I tell them "you have to be this much better" and it's a little trickier for women in the workplace because you've got to meet with the door open; be careful when you go to dinner at conferences. You just have to be a little bit more aware of how others view you as a group, whereas that doesn't happen if you're two men. I can go out drinking with two men and go back to the hotel at midnight. Nobody's 
going to bat an eye. You just have to be a little bit more aware, so I'm very aware of perception so my students don't get perceived in a different light. (Dr. Davis)

Other male faculty expressed how blatant the discrimination is and how they recognize it is unfair. No male faculty expressed this type of discrimination in a positive light and all acknowledged a desire to play a larger part in helping diminish it.

Oh there is absolutely a difference due to your gender. When it comes to science and meetings, the politics, all of it. It's just so, it's totally unfair. I'm never going to discount that there is some of what I do in reputation because of my perseverance. However, I do know that I can walk into a room and I can say some stuff and if you are a $5^{\prime} 4^{\prime \prime}$ not in shape female who was not very attractive, your voice is not going to be heard as much. It's absolutely true. Even if you have a way better idea. Your idea has to be twice as good as mine. There's definitely a larger barrier and it's totally unfair. (Dr. Thomas) In summary, gender is definitely a concern of biomedical research faculty at this institution. While some issues (such as meeting scheduling) can be handled internally, others (such as federal grants submission practices) are larger issues at a national level. What is encouraging is there is at least an awareness from both male and female faculty that those discrepancies exist and that they are not a good thing. Female faculty are also expressing that the environment for women in biomedical research is more positive now than it was when they started their careers.

Institutional type. Faculty at this academic health center were largely drawn to it because of the institutional type. Lower teaching loads, higher exposure to graduate students, proximity to hospital clinics, and available facilities all play a large role in 
attracting and retaining biomedical research faculty. One consistently positive trait was lower undergraduate teaching loads. "I think I was drawn towards the fact of not having a significant undergraduate teaching load. Not that I dislike teaching, but just knowing how much of a time commitment that aspect is when you go to a more traditional undergraduate type university, so I think that was part of it" (Dr. Anderson). Other faculty expanded on the appeal of lower teaching loads from experiences at prior institutions.

I think what drew me when I applied, so when I was at my previous institution I got sucked into a lot of student education. And I was teaching a lot and I had a grant and I felt I was hardly getting any research going because I had people coming and I had, like, 20 undergraduates one semester in the lab and it was just insane and three undergraduate thesis and there wasn't enough time for me because I was education. And so I thought I'm going to start applying to jobs where I don't have to deal with education [laughter]. And so that was one of the main reasons I came [here] because of the emphasis on research and yeah I mean that was it and then I got kind of sucked back in. (Dr. Grey)

While many faculty highly valued the nuances of working at an academic health center, there were concessions made that slightly reduced potential satisfaction. Although some faculty specifically work at academic health centers to escape larger teaching loads, others miss teaching undergraduate students.

You know I actually really like teaching undergrads and I feel like we miss out on that sort of, "oh my god!" moments. By the time they get to us as grad students they've had experiences as undergrads in research and they may have been a [lab] 
technician for a few years so it's less amazing to them. And I do enjoy teaching and I feel like that is something that is a strength of mine. (Dr. Anderson)

Faculty often cited partnering with undergraduate programs or participating in Summer research opportunities to fill that void. One faculty member took advantage of a unique opportunity and collaborated with undergraduate students in an entrepreneurship program.

We participated with like a project for two or three entrepreneurship programs where undergraduate students come in and then they started one here and they used us as their project material and that was kind of fun. They used us as their project material and even wrote up a business plan for us. So our first business plan was written by undergraduate students. So, that was kind of fun. (Dr. Black) Many faculty specifically mentioned the facilities offered at an academic health center that are unique and not found at other types of institutions. This availability of facilities often narrowed job searches to very specific institutions.

So a little bit of background I work on [a specific disease] and it is a very important global disease. It also requires specific resources in terms of a biosafety level 3 lab to do your research and so that can really limit the number of places I can apply. I can't do what I want to do at like [a liberal arts school] or even [public university] didn't have the facilities 5-10 years ago so that limited it. And I did look at sort of state schools as well as medical schools and the fit here was just a better fit than the other programs. (Dr. Anderson) Clinical translation was mentioned multiple times, which is the translation of bench science in a laboratory to eventually being administered as a therapy to patients. 
The symbiotic relationship between scientist and clinician is especially important to biomedical research faculty and mentioned many times as what drew them to an academic health center and this institution in particular.

Part of why I was drawn here is just availability for the type of research I do. Because what I do depends a lot on clinical translation, and so needing to be in close proximity to a medical school versus maybe a non-medical school, this was just where the opportunity was. So we were also being recruited to [another university] at the same time, but the proximity to the clinicians and interactions with clinicians and sample availability; you can get samples directly form the clinicians. That was a big draw here. (Dr. Black)

Access to clinicians and patient samples was frequently cited. Many faculty mentioned how it explicitly advances their work

In terms of really in particular in terms of my close association with the clinical aspect, the access to clinical samples, access to clinicians and surgeons and things like that really changes how you think about what you're doing. And we have had the opportunity to have access to material that my previous institution never really had, so it's really opened doors I think. (Dr. Parker)

In summary, faculty who work at this particular academic health center were largely drawn here because of the institution type. Lower teaching loads, access to patients from the clinical enterprise, and specific facilities all play a role in attracting biomedical research faculty and act as motivators adding positive value to their perceived job satisfaction.

In general, demographics are an important mediator when assessing job 
satisfaction and morale of biomedical research faculty. There are important differences in how satisfaction is expressed by female faculty, which is important to separate when assessing general satisfaction of a group of faculty. Also, aspects of this particular institution type (academic health center) can both increase and reduce expressed job satisfaction.

\section{Environmental Conditions}

The final category of mediators, environmental conditions, was especially important in terms of collegial relationships and student quality as motivators. Perception of administration and institutional climate were often demotivators, although these perceptions differ depending on how long faculty have been employed at the institution. Because environmental conditions are typically fluid in a work environment and most likely to change over time, there was a wide variety of responses.

Collegial relationships. All faculty interviewed stated that one aspect that brought the most joy to their work environment was their collegial relationships. Nobody spoke of negative relationships with their peers and all faculty mentioned a culture of collaboration and encouragement. Many mentioned that their relationship with colleagues was a primary reason for their continued desire to continue working at this institution. "The real strength here is the collaboration with other faculty. Despite all of this other noise, people really enjoy getting together to talk about science and share ideas and participating" (Dr. Johnson). Other faculty expressed similar sentiments.

I think [my relationships with faculty] are all pretty positive. I always tell prospective students or faculty that we're recruiting that [this institution] is just so collaborative that I really think it's a huge strength of the university. I don't know 
people that don't want to collaborate or talk to you. Some people get too busy, but it's not out of lack of interest or fear you're going to steal their work, it's just time availability issue. So I really think it's a positive relationship. (Dr. Smith) Other faculty reiterated the collaborative environment present with other faculty. Also mentioned was an environment where people felt safe discussing novel ideas without worrying about those ideas being stolen.

I don't think I've had any really bad relationships with other faculty, which is really nice. It definitely doesn't have that cut throat kind of competitive "I'm gonna steal your idea" kind of feel, which really allows more open and, throw ideas around and stuff like that. So I've found it really pleasant to interact with everybody. There are some strong relationships across different departments and some newer relationships that are just starting because somebody says "well why don't we think about the cross talk between our two programs and there's this mechanism that might give us money to do it if we think of something really cool" and that's the part where you get excited again because you're being challenged to think about your questions in a different way. (Dr. Parker)

Not only are collegial relationships highly valued, but they are also an important retention mechanism. Positive relationships with other faculty is one of the few unanimous qualities agreed upon by all faculty interviewed as a way this specific institution excels.

Student quality or relationships. Almost all faculty interviewed specifically mentioned working at an academic health center specifically because of the education mission. Faculty enjoy teaching, find meaningful value in mentoring, and consider high 
achieving student success an important part of their legacy as accomplished scientists.

I love working with graduate students. Whether it's my own graduate students and seeing their careers take off and see them catch fire on research and do well in their careers later. I just think that's the best feeling that I really enjoy. It's much more enjoyable than grant writing. I hate grant writing. (Dr. White)

Many faculty viewed mentoring students as part of their lasting legacy. Some of the faculty participants also came from families of educators or parents who were professors. I think that legacy is part of what I love. Like my dad was a professor and he had this big legacy he left and his legacy wasn’t his science, but his students. I still remember as a kid people coming to our house over the Christmas holiday and they were postdocs and former students who were professors now from all over the world and thank him for contributing to their careers and I always thought that was really the coolest thing that. I look at that and I don't really care about how many Science publications I've had or Nature publications; that's not going to be on your tombstone. It'd be really nice to leave this legacy of people whose lives you have affected in a positive way. I think that's more meaningful in terms of having something to look back on as an accomplishment. (Dr. Black) Additionally, many faculty mentioned maintaining relationships with their students far beyond their formal training period. Weddings were a theme mentioned many times. I always enjoy the mentor role. I've been to many weddings as the role of a mentor for this little transition in life, having like growing up parental input. I treat you like an adult and send you to amazing places and you're going to have to be the best and I'll always be here to help review some stuff. I love being in 
touch and being that longer colleague. Playing a little role and giving an opportunity. Making money only gets you so far, but collectively advancing something, I enjoy working with students who have ambition and just being there as a sounding board. My personal passion and satisfaction is the people. (Dr. Davis)

Some faculty mentioned that while they enjoy teaching, there is more that could be done to better the teaching environment or increase student quality. Many mentioned better supporting student funding as a way to increase faculty participation in teaching. I would like to see more support for student stipends so it's not a burden for faculty to take them on. Especially in this kind of funding climate, that's really a challenge. You have really young labs that are doing awesome stuff, but just haven't broken through yet to get that solid funding, so they can't take a student right now and that's a shame because those are probably the best labs for students. So, you know, if you could take some of that burden off of individual faculty, I think you could make that less stressful in the first place. Give students more opportunity and you could bring in more students. (Dr. Banner)

Other faculty expressed a desire for a culture change in the training environment. Newer faculty tended to express a lack of perceived holistic engagement that then disengages enrolled students. Many newer faculty wished for work environments where those conducting science would form closer-knit relationships and express greater levels of excitement. Instead, they view the environment as one where students and employees essentially put in their time and then go home.

So I think if we go back to what's missing here, I think that's what's missing here. 
That sense of a dynamic scientific community where all the way from the early trainees to the faculty who are just really excited about doing science and talking to each other about science. Here and maybe it's a bit of a west coast vibe too, it's a lot more like, "yeah well we do this during the day, but then we have to go do yoga and ride our bikes home," you know? It's different than other places. Where places like my post-doc lab, there'd be people there almost any time you could think of to go there and just, in other labs around us, just always working hard. No yoga, but then there'd be a big happy hour where the whole institute would come together and all the students and all the post-docs and I've never seen that happen here. Everybody's running to [get home], so that is missing. (Dr. Parker)

Although there is room for improvement, biomedical research faculty express high value and satisfaction from teaching and their relationships with students. Many extend that relationship with their students far beyond the initial training period.

Administration. Perspectives on administration at this particular institution were consistent. Faculty viewed their satisfaction diminishing over time, whereas newer faculty were either unaware of administrative decisions or were specifically recruited due to current administrative conditions at the time of their hiring. Faculty expressed a perception of basic science being of low value to the administration, which diminished their self-reported job satisfaction. Concerns were also expressed in terms of institutional priorities and salaries of top administrators. Specifically, many faculty noted that relatively high administrative salaries were perceived to be an institutional value regarded higher than stated institutional priorities - making administrative salaries a priority in 
itself.

A common notion expressed from faculty who had been at the institution for more than five years was a sense of nativity of institutional priorities. Faculty at the start of their careers focus on immediate tasks, whereas later on they begin to gain a sense of institutional perspective that often times does not align with what they had believed were institutional priorities.

I pretty much spent the first few years putting my head down and getting my research done. And so I've either buffered myself or was buffered by my chair and not involving me in too much politics. And then you pop your head up and go "what the hell is going on?" But to a certain extent I did not have any real significant dealings with administration or leadership. Like when you're junior faculty, you don't have tenure. You could piss someone off and you would be in a not good place where as a tenured full professor can sort of say things and approach the leadership. And so I think my response is I try not to notice. (Dr. Anderson)

Senior faculty often describe their perception of administration as declining since their arrival with more supportive administrations having existed closer to when they first arrived.

Ironically the most support from the administration we ever had was when I first got here. The Dean valued basic science. [The Dean] valued what the Chairs were doing at that time to build up basic science and in hindsight he was the only one who has ever done that. The university has obviously changed dramatically and drastically. The administration switched from being a support organization to 
being the reason why the institution exists. Basic science changed from a core value of the university to a financial liability. (Dr. Johnson)

A common demotivator mentioned by most faculty regarding administration was a lack of transparency or understanding of institutional objectives. Finding out about institutional information via public newspapers was commonly shared. Dr. Grey, for instance, shared her perspective as faculty who have been employed for more than 15 years.

I think I've got more of a negative perspective on the administration. And you know I think that's more of about information sharing and you're sort of there working hard and you read in the [local newspaper] that someone is earning four times more than you do and you think I'm working really hard, I bet I could do their job. And is what they're doing worth four times more? So I think that fosters sort of negativity and I think you get more cynical with age, but with politics you realize that people are not necessarily doing things for the good of the students or the faculty or whatever there's a self interest that while you hope is fairly small, data suggests it is not as small as you would like. (Dr. Grey) Another faculty concern was interpretations of administrative objectives that diminish the fundamental science they conduct. This usually related to current fundraising objectives, but also national trends in funding priorities.

I guess I would say the one way I'm on the fence and that may be part of my misunderstanding of what [the administration] really stand for. My impression towards this push of everything needs to be applied and translational that even though as a medical center we should strive to do that, to say it that way de- 
emphasizes and de-values what basic research can do and we should never do that because we $100 \%$ need basic research all the time and it's the people that are studying some bacteria that we think doesn't matter at all is the discovery that allows us to do something absolutely amazing, right? So the balance in how we communicate that priority can be very discouraging who value basic research. And that's not just the messaging, but how it feels with money and space is getting allocated and things like that. I understand some of that shift, but I think it could be better balanced. (Dr. Banner)

One specific source of administrative frustration is a lack of participatory leadership. Faculty often cited examples of being involved in committees that feel more like disingenuous attempts to feign input, but are largely ignored. Committee work was brought up as a demotivator multiple times not because of the committee itself, but because of the perceived lack of genuine contribution.

The one thing I find a little bit, and I don't think it's unique here and has probably been the case at every place I've been, but transparency in terms of what are our goals and what are we trying to do is really lacking. Administration will act like they're asking your opinion and put on some meeting where you come and write things on a board and then you never hear of it again. So it just starts to feel like an exercise so that I feel like I'm involved, but I don't really need that. You can tell me I'm not involved and I'd actually prefer that than this show that wastes my time for really no reason. So that I think is a frustration of mine that I've seen always and it works its way all the way down the chain. And that's the part of the job where if you can't see if it's changing anything, it really feels like it's pulling 
you away from what's important and that's really frustrating if you can't say no.

(Dr. Martin)

This feeling was expressed by other faculty as well. Dr. Miller expressed further frustration with committee participation that leads to results not endorsed by the committee.

Having committees and ignoring what comes from those committees is a source of frustration for me. Once again a committee was held and all of a sudden what is moving forward isn't at all what came out of that committee. There were strong dissenting voices from that committee and those will continued to be ignored. I anticipate the same basic thing will happen. The administration will shove their ideas down our throats because they can because they have all the power. (Dr. Miller)

Not all perceptions about administration were negative. Newer faculty expressed high levels of satisfaction with current administration. Many junior faculty specifically named administrators and department leadership as the reason they were recruited and ultimately accepted the position.

The biggest part of my recruitment though was the leadership here. [My Department Chair] being here. Recruiting people that really worked in my specific field and sort of building a team of people that would be really synergistic with the types of things I thought of was really the biggest thing. And since coming here I think I probably didn't even appreciate the resources that I would have as a result of being at a health center. The way I conduct my research now is quite different than how my previous bosses did. (Dr. Parker) 
Devaluation of basic science, unclear institutional mission, and frustration with participatory leadership were expressed as main points of concerns regarding administration. While new faculty pointed to current administration as a positive value, faculty with longer tenures pointed to larger system-wide issues as sources of dissatisfaction.

Institutional climate or culture. Comments about institutional climate and culture largely centered on the perceived business model of the institution and prioritization of the clinical enterprise. Some also felt that the institution no longer resembles a university. Dr. Johnson expressed the following:

It has been said this university lacks the tradition of being a university and that's the source of many woes. There is no administrative understanding of being a university, but we're a biomedical corporation or a hospital corporation with schools tacked onto the side. I see no reason to see why that's going to change. Academic health centers inherently have both clinical (more business-focused) and research missions, although faculty at this institution felt that the balance between the two is not in alignment.

I think my perception is they still want to run this university as a business and it's been that way since I got here years or so ago and I think that's the wrong way to run an academic institution. Obviously we need profit to make it sustainable, but you can't just run an institution of learning in higher education that way. Profit can't be the purpose. So there are ways you have to be able to separate that out. And so I feel like there's this lack of commitment, and this sounds kind of corny, but to the higher purpose of education and training. And it's all about the bottom 
line here and it always has. (Dr. Black)

While access to clinicians and clinics was previously mentioned as a motivator, the administrative focus on the clinical enterprise was perceived to be out of balance with research. Many faculty expressed concerns of too much clinical focus being detrimental to the rest of the institution's missions.

I think this is something that is pretty global and not specific. It has gotten better, but I don't think it's 100\%. [This institution] has such a huge clinical enterprise that in the past it didn't seem that research was very valued by the university. I think that has improved. I think creating an appreciation for the research that is done and understanding that while they might not see the exact line item contribution financially that research brings them, it does increase the stature and knowledge and reputation of [this institution] that can lead to some greater dollar amount if that's their interest. (Dr. White)

Not only does the perception of focusing too much on the clinical mission decrease job satisfaction from faculty because of its internal consequences, but others feel focusing on the clinical enterprise also hurts the institution's broader reputation.

So here the fact that still remains when they get nervous they go to a local hospital model and an unwillingness to invest in any of the infrastructure that is absolutely required to enhance your education portfolio. And there's only so much you can do if you want to get on the national scene. That is a failure to invest in the infrastructure, that's fatiguing. If they said, "we don't want to do that" then fine. But if they say, "we want these things to happen" and yet are unwilling to invest, that is my frustration here. If you just want to be a local hospital then be a local 
hospital. (Dr. Thomas)

The institutional climate and culture at this institution is largely perceived to be out of alignment with the priorities and values of biomedical research faculty. Faculty participants unanimously mentioned distaste with the corporate environment as well as a perception that administrators held a low value for the research biomedical faculty conduct.

\section{Triggers}

Triggers are fundamentally about change. All faculty will experience change throughout their careers and any change whether personal or professional has the potential to affect how they perceive job satisfaction. Change in itself can be a source of stress both positively and negatively. While responses in these interviews did not necessarily align with Hagedorn's sub-categories of change, change as a broader concept was expressed as constantly fatiguing and detracting from job satisfaction.

The single most talked about source of job dissatisfaction was the concept of stability. All faculty interviewed used the exact word "stability" to describe different aspects of how instability decreased their job satisfaction. Faculty interviewed expressed feelings of change fatigue as well as misalignment with institutional changes and their own research. While the triggers, or sources of change, were not those identified in Hagedorn's model, change for biomedical research faculty largely fell into categories of funding, retention, and space.

\section{Funding}

All faculty interviewed expressed lack of funding stability as a source of stress. While it has already been acknowledged that how a salary is derived is a source of stress, 
the larger issue at hand is lack of stability in reliable funding. Many faculty expressed desire for the institution to do more to help with stability through hard money or greater support and understanding for how federal grant dollars work. The new faculty contract has unanimously been reported as helping with that issue.

At one point we had a financial model where we had bonuses and things like that and my department and I were competitive with that, but really what was more important was having stability. What the new contract now does is give you that stability rather than that potential for bonuses. We're not in this for the money. If we were we would all be in industry. And so really we just wanted some stable model that lasted, so I think that has improved faculty morale a lot because it was so nerve wracking before. Just knowing you have that amount you can say "ok, now I can add to it" and know you have it. (Dr. Smith)

Also shared were feelings that the institution could be doing more to add stability to faculty funding. The lack of funding stability creates what many faculty expressed was a sense of job insecurity due to the continued need to seek soft money.

I think one of the things that is never captured or appreciated from especially people from outside of what we do is the issue of job insecurity. So like this last HR survey they asked you, “is your job stressful?" Well yeah it's stressful, but you didn't ask me why it's stressful. And for me the most stress is the uncertainty of the funding. We were talking about having secured funding for students so you're not having to pay their stipend, but wouldn't that be great if you could do that for faculty? And how much happier and more effective we'd be if we weren't spending our time writing grants for $50-60 \%$ of our funding all the time. 
(Dr. Black)

Inequalities in funding opportunities were also frequently expressed. Faculty who felt they could argue that their research aligned with an institutional priority found it easier to obtain funding even if their projects were less exciting than their peers' projects.

Well I think right now the biggest source of job dissatisfaction is probably stability and the funding climate. And again that sometimes splits across this basic/translational line, which is also very frustrating. More and more people who are able to twist (and I fell into that category) who can twist it and make it seem like it's going to be a big deal for, say, cancer, we get funded with a scientific premise that isn't any more exciting really than the basic immunologist sitting next door. It's kind of demoralizing and obviously there's limited funds and things like that, but somehow, somehow our system needs to reward productivity and strong science over some theoretical impact on cancer patients in 30 years. Because the reality is most of what we're doing is not going to cure cancer in 5 years. But it doesn't mean it's not really important work to be doing. (Dr. Parker)

Only one faculty member explicitly stated that he enjoys the current funding climate and finds that it adds job satisfaction. This individual saw the current climate as an ideal opportunity to grow and further stand out.

I really enjoy [grant writing] too. I love the gamesmanship over grant writing. It's a really great activity for me. It's really tangible. You either get the money or not. Because the science, how relevant and exciting your results are, there's a little bit of politics, there's timeliness, even if I discovered the greatest thing about 
(whatever) is it going to be on the cover of an esteemed journal? How do you rate the quality of science and weigh it compared to one field over another field? But with money it's just money. You either get the money or not. (Dr. Davis) Aside from Dr. Davis, changes in funding stability were perceived as a constant stressor. Faculty expressed that more hard money available from the institution to help with changes in soft money funding over time would decrease stress and lead to more productive scientists. This was also expressed as an issue much larger than just this institution and something that colleagues at most other institutions deal with as well.

\section{Retention}

The other category of change that faculty expressed as a source of stress is that of retention and incentivizing their colleagues to continue their employment at this institution. Many reported a culture of a revolving door where colleagues and faculty are constantly leaving the institution. Because science is a collaborative effort and faculty derive much of their job satisfaction from colleague interactions, frequently losing colleagues was reported as a great source of stress and overall job dissatisfaction.

One thing, it's just a minor thing here, is how they about the grant writing and social dynamics. A lot of people come here and they're successful and they're out. Up and out kind of things. It's hard to retain people here unless they work with great colleagues like I do. And the reason being is we definitely have a doom and gloom type of communication. (Dr. Davis)

One thought on why faculty retention is poor is due to mismatching institutional priorities. This idea was mentioned multiple times for both why faculty felt their colleagues left and why faculty themselves have considered leaving the institution. 
The university and administration seems to get tunnel vision and they focus on one thing and they do that to the detriment of the rest of the $80 \%$ of the institution. If you talk to more of the senior faculty they'll say that this is like a repeating pattern that [this institution] seems to do in terms of just getting singled minded on something. And that doesn't reflect well on the entire institution, but it also I think it probably impacts our ability to retain solid people in certain areas. (Dr. Anderson)

Because collegial relationships are so important to increasing job satisfaction for faculty, watching their peers leave for other institutions is a detrimental demotivator. All faculty interviewed acknowledged the culture of poor faculty retention, but none blames their colleagues for leaving nor did they believe the revolving door culture was likely to change.

\section{Space}

Finally, some faculty mentioned the space they work in as an issue. While it is known that faculty who receive diminished grant dollars often see their labs shrink, others like Dr. Grey describe an environment where they have to fight to keep their space in the face of competing institutional priorities.

I think this is sort of related to the hard money thing, but I think stability would help. I understand that administration don't want to create stability because maybe people will rest on their proverbial laurels and become dead wood and not do anything, but things like defending my lab space--I have lab space [in a campus building] and they're trying to get my lab out of there. I mean I have a grant to run the research that I run, but they want me out of the space so that they 
can put clinicians in the space. And the only thing that stopped that happening so far is they have no idea where to put me and when I came they built it specifically for my research and nobody wants to pay for recreating that somewhere else.

They reduce job satisfaction because you don't feel valued because you're not valued. Your space might be valued more than you are. (Dr. Grey)

Although stability in funding, faculty retention, and space are not sub-categories of triggers in Hagedorn's model, they all meet the general principle that triggers are changes and significant events that impact job satisfaction. These are concerns shared by almost all faculty and were consistent across gender and length of employment.

\section{Conclusion}

Biomedical research faculty interviewed at this institution express great satisfaction and value in their work. They enjoy the collaborative nature of their work and the relationships they have with colleagues, mentoring and teaching students, and the institutional type is well suited for the type of research they conduct. Areas to improve on job satisfaction largely revolve around institutional priorities, perceptions of participatory leadership, and an overall sense of lack of stability. While many faculty spent more time talking about what they would like to see improved, almost all were quick to reiterate that they do enjoy their jobs and are overall satisfied with their work. 


\section{CHAPTER 5: Discussion and Implications}

The purpose of this study was to explore the personal and professional factors that contribute to positive morale and job satisfaction for biomedical research faculty at an academic health center. Twelve faculty were interviewed and their responses were coded according to Hagedorn's (2000) Conceptual Framework for Faculty Job Satisfaction. Those responses brought forth six clear themes that indicate aspects of faculty worklife that both add and detract from job satisfaction: (1) faculty enjoy science and mentoring, (2) funding is an issue (but not salary), (3) relationships matter, (4) faculty feel little institutional identification, (5) female faculty feel discrimination, and (6) length of employment influences job satisfaction and morale.

The most promising result from this study is clear; biomedical research faculty at this one academic health center genuinely enjoy the work they do. Scientific research brings them a great sense of personal enjoyment, mentorship brings a sense of fulfillment, and their collegial relationships with other faculty keep them motivated to continue asking new questions to investigate. What is also clear is that there are other aspects of the work environment that detract from what could be a more satisfying role. Some issues are large and might be best addressed on a national level, but others can easily be addressed in some way at an institutional level. This chapter presents the assessment of the conceptual framework in the context of the study data, answers to this study's research questions, addresses possible implications and recommendations for academic health centers, and concludes with recommendations for future assessment and research. 


\section{Conceptual Framework}

Responses in this study aligned closely to Hagedorn's (2000) mediators, but not as strongly to the framework's designated triggers. Instead, all participants brought up the concept of stability as a source of decreased satisfaction. This sense of stability was often in relation to funding mechanisms or institutional policies, which are not explicitly outlined in Hagedorn's (2000) framework (see Figure 2).

\section{Mediators}

Stability Triggers

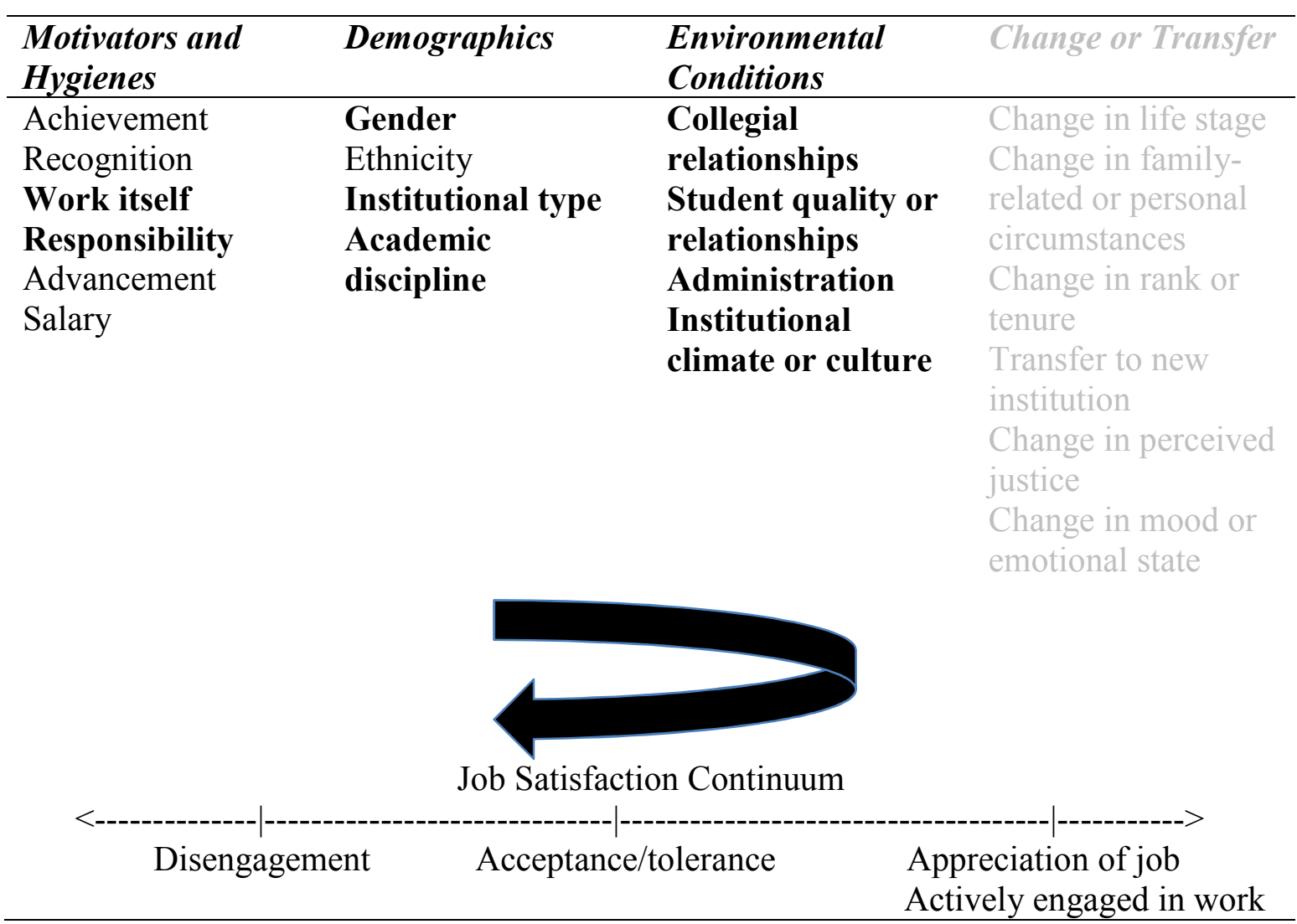

Figure 2. Hagedorn's (2000) conceptual framework of faculty job satisfaction adjusted by findings in this study. Items in bold were found to be strongly valued by biomedical research faculty, while those in grey were not found to align closely with job satisfaction. This study was unable to assess the impact of ethnicity due to the sample size. Adapted from framework by Hagedorn, L. S., 2000, Conceptualizing faculty job satisfaction: 
Components, theories, and outcomes. In L. S. Hagedorn (Ed.), What contributes to job satisfaction among faculty and staff, p. 7. Copyright 2009 by Jossey-Bass.

This study also found that Hagedorn's (2000) Job Satisfaction Continuum is too simplistic for biomedical research faculty. Instead, faculty report different levels of satisfaction depending on different aspects of their job. For example, a faculty member might fall at "actively engaged in work" on the Job Satisfaction Continuum when talking about their role as a scientist, near "acceptance/tolerance" in their capacity as an educator, but "disengaged" in their relationship with the institution. Therefore, Hagedorn's (2000) mediators were closely aligned to responses, the Job Satisfaction Continuum works best when assessing individual roles, and triggers could be replaced by stability as a better way to gauge change.

\section{Research Question 1}

How do biomedical research faculty at an academic health center describe the mediators and triggers that influence their morale and job satisfaction? Biomedical research faculty described many mediators and triggers throughout this study's interviews. Out of those descriptions, four common themes emerged. This section explores how biomedical research faculty described funding, collegial relationships and faculty retention, identification with the institution, and the conducting biomedical research (work itself).

\section{Funding}

Faculty are not motivated by salary, but highly value funding stability. This preference was expressed by all faculty interviewed who stated that changes in institutional funding models and federal sources of grant funding decrease job 
satisfaction. This institution's recent contract negotiation to cover at least $50 \%$ hard money helps, but there is still a great amount of concern regarding institutional funding and what that funding reflects in terms of how the institution values its research faculty. Faculty understand that supporting their research with soft money is a reasonable expectation, but too much reliance on soft money also has the effect of making them feel like independent contractors. This finding is consistent with existing literature regarding how the quality of benefits and services influences faculty job satisfaction (Johnsrud \& Rosser, 2002).

While participating faculty did not cite salary itself as a source of dissatisfaction, they did mention that differences between colleges, departments, or how funds were distributed was a source of decreased job satisfaction. For example, multiple faculty mentioned that they often read local newspaper articles disclosing the salaries of top administrators and felt like their own lower salaries indicated how little they were valued by the institution. No faculty mentioned a desire to obtain a higher salary, but instead expressed feelings of decreased job satisfaction through perceived inequality. These concerns are more aligned with differences in perceived justice. While salary and compensation are at the root of the issue, the actual problem is perceived inequality or justice, not salary itself (Johnsrud \& Rosser, 2002).

Ultimately, salary is not a source of job satisfaction nor does it decrease satisfaction. Faculty want to feel that the quality of their salary and benefits is distributed consistently and in line with the institution's stated values. Frequent changes in financial models, disparities in salary between peers and departments, and disproportionately large administrator salaries all lead to decreased job satisfaction. 


\section{Collegial Relationships and Faculty Retention}

There are many reasons why might choose to leave an institution and seek employment elsewhere, but at this institution stability in employment retention is highly valued by faculty. As described earlier, faculty at this institution highly value their relationships with colleagues and those relationships bring with them high levels of reported job satisfaction. What many faculty have noticed, however, is that their colleagues seem to be leaving the institution and seeking employment elsewhere at a greater than reasonable rate. It was also stated that many faculty left not necessarily for career advancements, but that many were taking lateral moves or demotions due to displeasure with the institution. In regards to the findings of this study, this is important because faculty leaving the institution decreases the job satisfaction of remaining faculty due to the loss of their valued colleagues.

The importance of collegial relationships is consistent with existing literature. Opportunities to collaborate and be a part of an academic community increases job satisfaction and faculty report higher levels of morale (Bozeman \& Gaughan, 2011; Hagedorn, 2000; Nyquist et al., 2000; Sabharwal \& Elizabeth, 2009). This study’s example is a bit different, however, in that faculty report very high levels of satisfaction in terms of opportunities to collaborate with their peers. What brings this group dissatisfaction is the high level of perceived turnover. Existing research explains why faculty choose to leave their jobs (Johnsrud \& Rosser, 2002), but not necessarily what that does to the job satisfaction of those they leave behind. This study shows that faculty turnover does in fact impact job satisfaction of faculty who stay and is something that should be further studied through future research. 


\section{Identification with the Institution}

Faculty who report identities strongly tied to their institution have been found to have higher self-reported morale and job satisfaction (Rice \& Austin, 1988). This study's participants, however, reported little or no identification with the institution. While participants identify strongly with their closest peers and their department, there is a distinct gap between their own area of research and the institution's larger goals. This lack of congruency between one's disciplinary career (described as strong) and institutional career (described as lacking) speaks to the core of both a lack of institutional identification as well as organizational momentum (Rice \& Austin, 1988).

One source of this lack of institutional identification largely came back to the issue of hard and soft money. The less this institution funds its faculty with hard money, the less faculty value their relationship with the institution. Almost all participants used the words "independent contractor" to identify how they feel and as such, their employment status. Dr. Thomas summarized this, "Less than half of my actual time is covered. There's no financial investment or commitment from the university level to me." The dual soft/hard money funding dynamic is not unique to this institution, so this dynamic may be relevant to many academic health centers and could be a source of job dissatisfaction as well as diminished institutional identification.

While synergy with the clinical enterprise is vital to the research of biomedical researchers and is highly valued, that relationship is also a source of dissatisfaction that is detrimental to researchers' institutional identification. When institutional priorities focus primarily on the clinical enterprise, biomedical research faculty feel less valued, which leads to less institutional identification and decreased job satisfaction. Some faculty 
expressed that they feel there is a greater appreciation for specific types of research (such as cancer research), but that research in general is a distant priority in comparison to generating clinical revenue. That said, almost all faculty expressed an understanding of the importance of clinical revenue and how ideally there would be a balance between the clinical enterprise and research. This too is supported by literature regarding academic industrial activities (Bozeman \& Gaughan, 2011) as well as scientists feeling like second class citizens at academic health centers (Cole et al., 2009; Holleman et al., 2014).

\section{Conducting Research and Work Itself}

Biomedical research faculty participating in this study genuinely enjoy the work they perform at their academic health center. What initially attracted them to scientific research continues throughout their careers and they stay because of science and participating in education. When asked what continues to motivate them, all faculty mentioned that conducting science is still a rewarding and exciting endeavor. Most others also mentioned that they especially enjoy mentoring graduate students and find training the next generation of scientists part of their legacy. While projects change, students come and go, and institutional priorities shift, biomedical research faculty are driven throughout their entire careers by a fundamental curiosity and drive to improve human health. How faculty experience the core functions of their job is supported by literature as a primary means of job satisfaction and is true at this institution as well (Hagedorn, 2000; Rice \& Austin, 1988).

\section{Summary}

Faculty describe the work itself — research and education-as a major source of job satisfaction. They also derive job satisfaction from an environment that is conducive 
to rich collaboration that further enhances the work environment. What decreases job satisfaction are issues related to funding, faculty retention and turnover, and a lack of identification with the institution. Less is currently known regarding issues pertaining to soft money funding mechanisms as well as how turnover impacts existing faculty collaborations.

\section{Research Question 2}

What are the differences in in morale and job satisfaction of biomedical research faculty by gender and length of employment at their current academic health center? This question is best answered by two main themes that emerged from the data. The first theme is female faculty express different concerns than their male counterparts. The second theme is faculty who have been at the current institution for more than ten years express different concerns than faculty who have been there for fewer than ten years.

\section{Female Faculty}

Female faculty at this institution continue to face discrimination in biomedical research. Furthermore, their male counterparts are aware of the discrimination. Senior female faculty agree that things are getting better and more women are in leadership roles, but as supported in previous literature, others have noted than many female faculty in leadership roles get there by acting like male leaders (Kezar, 2014). All female faculty mentioned the need for "self-promotion" and how they are uncomfortable with it, but acknowledged that it is necessary for their female colleagues to be promoted or obtain leadership roles. While it is promising to hear that progress has been made and that current female faculty are not facing the type of discrimination their more senior peers have, it is clear that there is room to improve gender equity. 
It also continues to be a challenge for female faculty to manage family and worklife balance. All female faculty interviewed mentioned some aspect of their job as being unfriendly towards faculty with families (specifically young children) or their job as being the reason they ultimately chose not to start a family. While all faculty expressed feelings of having to constantly stay productive in the lab and maintain funding steams, female faculty expressed frustration with the perceived inability to temporarily take a break to raise children. These feelings are consistent with what has been reported in previous research specifically about women in STEM fields trying to raise families and the difficulties they face in the workplace (Sabharwal \& Elizabeth, 2009; Wolf-Wendel \& Ward, 2014). Previous research has also found that "[female faculty] with children published less, had slower self-perceived career progress, and were less satisfied with their careers than were men with children" (Sabharwal \& Elizabeth, 2009, p.524). While this study did not look at rates of publication, many female faculty interviewed mentioned that their career trajectory was slowed down or halted due to having children. Starting a family or having to adjust worklife due to changes in family-related or personal circumstances is also noted as a potential trigger in Hagedorn's conceptual framework (Hagedorn, 2000, p.7).

Female faculty feel like progress is being made in terms of women in leadership roles, but still feel there is much progress to be made to make the work environment more equitable. Many feel they have to act in ways they do not feel comfortable (such as self promotion) to get ahead. Female faculty also feel that having children is a choice that is often detrimental to their career trajectory and not fully supported by their work 
environment. Both of these are issues that academic health centers can explore further to determine how to approach at their own institution in order to increase job satisfaction of female biomedical research faculty.

\section{Length of Employment}

This study originally planned to look at faculty responses by rank, but what ultimately makes a larger difference in perceived job satisfaction is length of employment. Because biomedical research faculty do not always fall under promotion and tenure guidelines that follow strict timelines, the faculty at this particular institution indicated that academic rank meant very little in terms of career length or seniority. Literature on faculty morale and job satisfaction indicates that rank plays an important role in determining what influences faculty throughout their career (Eyupoglu \& Saner, 2009; Johnsrud \& Rosser, 2002; Rosser, 2005; Seifert \& Umbach, 2008; Wolf-Wendel \& Ward, 2014), but that did not hold true at this institution. Instead, after about ten years, a theme emerges where faculty have lower opinions of administration and leadership than their newer counterparts. Conversely, newer faculty typically cite that they were recruited because of current leadership and are on board with current institutional objectives. As those institutional priorities or directions change, more senior faculty tend to not align their values with newer visions.

For example, faculty who had been employed at their institution for more than ten years often referred to the institution's public fundraising efforts as a waste of time or something that they did not understand fitting with the institution's other goals or missions. They also felt like their opinions did not matter in pursuit of these new initiatives. Instead, the common sentiment was that the university would choose an 
agenda and push it without regard to whether existing faculty supported it or not. Faculty employed less than ten years, however, often described those same initiatives as to why they accepted their position in the first place.

Another example of disengagement is in regards to the institution's education mission. Faculty employed longer than ten years felt that although they were often the ones who developed existing curriculum, they had little say in conversations about anticipated changes to graduate education. Many felt that their graduate programs were fine they way they were and required no major overhaul. Faculty employed less than ten years, however, often described ways in which they think graduate education could improve and were more welcoming of proposed changes to the institution's graduate education model.

In regards to Hagedorn's conceptual framework, most of the expressed complaints about current administration had to do with changes in administrators themselves. Past Presidents, Provosts, or Deans were usually regarded more positively than current ones due to changing perspectives on institutional priorities or policies. While the conceptual framework can account for this as changes in environmental conditions or a trigger of change in perceived justice, ultimately it appears that length of employment can negatively impact job satisfaction (Hagedorn, 2000). Due to the rapidly changing environment of national healthcare reform, academic health centers will always be institutions experiencing change. How academic health centers respond to that change and communicate to their faculty how and why they adjust has the potential to increase job satisfaction for faculty who have been employed longer. 


\section{Summary}

Female faculty express issues of job satisfaction differently than their male counterparts. Faculty of different employment lengths also express satisfaction differently depending on how long they have been employed. Both of these issues are important in considering how institutional priorities and policies can impact faculty job satisfaction. While issues of satisfaction by employment length may relate more to shifting institutional priorities or missions, issues pertaining to female faculty job satisfaction are largely consistent and have been slow to change over time.

\section{Recommendations for Academic Health Centers}

Biomedical research faculty at this institution enjoy the work they do and their collaborative environment. Those two aspects lay a foundation for improvement. Each of the following recommendations could build towards increasing job satisfaction at this institution. Based on responses regarding job satisfaction from this study, it is recommended that institutions implement efforts to talk with their faculty, provide funding support, enhance faculty retention efforts, increase faculty identification with the institution, provide greater support to female faculty, and increase faculty/mission alignment.

\section{Talk to Faculty}

Before an institution implements any changes, it is recommend to first talk with one's faculty. Faculty participating in this study often noted that they are never asked about their work or their opinions. Dr. Johnson specifically noted that the first step to fixing faculty morale would be for the institution to identify that faculty morale is important. Taking the time to talk with faculty about their jobs, their worklife, and what 
matters could go a long way in determining the most important issues. It could also offer an opportunity to identify relatively small issues to could be addressed easily and quickly. Small efforts to improve job satisfaction can make a large impact and build good will. Without taking the opportunity to talk with faculty, though, any solutions implemented may fail.

\section{Provide Funding Support}

One recommendation for both this institution and other academic health centers is to implement a system of gap or bridge funding (funding available to faculty between the end of one grant and the beginning of another) that is both considered fair and accessible for biomedical research faculty. Because these faculty base their salary and research on multi-year grants from funded by external agencies with revolving agendas, it was expressed many times that it would be helpful if the institution could provide additional stability for years with dips in grant or other external funding. The institution in this study does have gap funding, but the criteria one has to meet to apply requires one's funding and research capacity to be so diminished that it is the equivalent of starting over from scratch as noted by Dr. Black:

The length of time it takes to get you've already had to let everybody go and you're basically starting training a new staff and you recruit and it takes another six months to get up and running again. It's just a really inefficient way to do science.

This approach, while made in good faith, is too little too late. A more proactive approach that bridges funding gaps before they become catastrophic could reduce instances of lab shutdowns as well as potentially save more money in the long run due to fewer 
disruptions and lower turnover.

Another way of addressing the issue of funding support is to adopt more of a team or institute approach to funding. Instead of funding being tied specifically to one person, some suggested pooling funding across multiple researchers to better weather

inconsistencies in funding streams. This could mean instead of setting up faculty-specific labs, faculty could get together and share space, equipment, and even lab personnel. This might also mean finding ways to incentivize the research and clinical missions to work more closely together instead of operating in separate financial silos. This may not be feasible for all faculty or disciplines, but is one suggestion that may be less costly and was spoken of positively by participants in this study.

\section{Enhance Retention Efforts}

While it is not feasible to completely eliminate faculty turnover, faculty retention at this institution is not perceived to be an institutional priority. Because the perception is that the institution does not value faculty enough to retain them, many faculty believe neither they nor their colleagues matter. From an institutional standpoint, simply doing exit interviews, understanding why faculty leave, and acknowledging those reasons could go a long way toward decreasing faculty turnover. Although faculty will always come and go, faculty turnover can be detrimental to scientists whose work is often planned years in advance (both the experiments they conduct and the grants that fund them). Having a faculty member resign results in decreased job satisfaction of their colleagues who remain. Because this issue also impacts the job satisfaction of those who remain, it is recommended that institutions understand the core reasons for turnover and how they can 
improve the work environment for those who stay.

\section{Increase Identification with Institution}

Because all faculty interviewed mentioned funding as an issue that decreased their job satisfaction, this is an important area that the institution could address. While it is likely unreasonable to expect full funding of all faculty with hard money, a balance needs to be struck if the desire is to have their faculty be on board with institutional goals or objectives. As it stands, faculty who feel like independent contractors or interpret their research as inferior to the clinical enterprise are not likely to support or feel motivated to care about institutional objectives or goals. This feeling can lead to less general service to the institution, decreased willingness to collaborate, increased feelings of internal competition, and general indifference to the institution itself (Rice \& Austin, 1988).

While funding often came up as a reason for lack of institutional identity, many faculty also felt that they simply did not understand the priorities of the institution. Many expressed that the institution's objectives felt hyper-focused on one issue without communicating how that would benefit the institution or how faculty could become involved. Dr. Andersen's comment about the university ignoring $80 \%$ of the university to focus on the other $20 \%$ was a common sentiment in how faculty often felt ignored or not important. Institutional objectives will always change, but academic health centers choose how they communicate that change and may need to adjust how they communicate to biomedical research faculty. Better communication of institutional priorities, how faculty can be a part of that, and why it is important could go a long way toward increasing faculty identification with the institution. 


\section{Support Female Faculty}

There is an opportunity for institutions to adapt policies in order to support faculty (especially female faculty) who wish to start families. One issue in particular that would be a feasible starting point is modifying policies and practices around scheduling meetings. Dr. Martin, for example, shared how in her department she often misses out on workgroups or meetings because they are scheduled before or after more typical work hours. She also mentioned how this was less of an issue at a previous institution she worked at due to a greater organizational value in holding more family-friendly scheduling practices. Shifting scheduling priorities to fit within more typical business hours does not mean banning meetings outside of those times, but should include meetings that are held at a certain regularity (e.g., weekly) or require a certain number of people in attendance (e.g., a Department-wide meeting). Reducing instances where faculty with families have to choose their family over meetings could be interpreted as a large gesture towards inclusiveness and increase job satisfaction for faculty with families.

A more complicated, although equally important next step would be to implement institutional policies or resources that address biomedical research's requirement for constant outside soft money. Dr. Thomas succinctly stated that science doesn't care if a woman takes a break to have a child, but it's the competition and expectation for funding that never takes a break. One solution could be to provide temporary hard money to support faculty salaries while taking short-term leave to support the birth or adoption of children. Temporary support such as this would remove the stress associated with taking funds away from the lab and potentially decrease the feeling of the need to get back to work as soon as possible. It could also temporarily offset the amount of time needed 
before having to submit a new grant. Similarly, institutions could also better support the research mission in general by providing a more proactive administrative support model that not just manages grants obtained, but assists with location and applying for new funding opportunities. Either of these concepts could provide an immense amount of additional support to faculty with families while also reducing the nation-wide pressure to constantly produce and not lapse in soft money funding.

\section{Increase Faculty/Mission Alignment}

Institutional fit is subjective and academic health centers are not static environments. New faculty are recruited and attracted because of current conditions and older faculty may leave because of changing conditions. Academic health centers will always experience change in administrators and objectives, but what would help support change is to be in tune with how changes impact current faculty and what institutional supports can exist to help them. The faculty in this study often had an unclear understanding of institutional objectives and were not sure which activities were priorities. This especially held true for faculty who had been at the institution the longest as they expressed losing track of which issues remain priorities. Dr. Black gave the following example:

I really fear [the institution] doesn't have the vision to see forward the sustainability of some of its ideas, but that's easy for me to say because I'm not responsible for those decisions. I can say, "well that was weird why are you spending so much money on a building when we don't have anybody to put in it?" It's like we start something with no vision and then move to the next. It's so frustrating. 
Clearer and transparent communication of institutional objectives, opportunities to meet new administrators, and assistance to help faculty succeed in meeting those new objectives could decrease faculty turnover. Faculty who align with institutional objectives express higher job satisfaction and morale, so it would be beneficial to focus on those who were recruited under different objectives (Rice \& Austin, 1988).

\section{Summary}

Some of these recommendations may require more time or resources than others, but all could play a part in increasing faculty job satisfaction. None is meant to be a panacea, but any one recommendation could increase the satisfaction of faculty. What is important in increasing job satisfaction is to identify a first priority and an implementation plan. Making continuing efforts to improve job satisfaction along with seeking faculty input could help towards improving overall job satisfaction.

\section{Future Research and Assessment}

Although this study only explores how 12 faculty express job satisfaction at one institution, its results show that there are unique differences in how faculty at one academic health center express job satisfaction. Potential areas for future research would include ways to improve faculty identification with the institution, broader surveying at additional academic health centers, how academic health centers can support female biomedical research faculty, how soft money impacts faculty job satisfaction, and exploring what happens to faculty who remain at an institution after high instances of faculty turnover. Any one of these studies could assist greatly in further understanding job satisfaction for faculty at academic health centers. 


\section{Assessment at Interview Site}

Recommended future assessment at the site of these interviews would include investigating ways to better help faculty identify with the institution. Female faculty expressing lower job satisfaction, faculty feeling like independent contractors, and low morale due to faculty turnover have made participating faculty express low identification with the institution. While these issues would likely need to be explored separately, they all contribute toward the general lack of institutional identification.

It is also recommended that the interview site tailor its future surveying to its employees by mission-type. For example, what is important to biomedical research faculty might impact job satisfaction differently for physicians or public health faculty. Faculty in this study expressed feelings of being insulted by the previous employee survey or that the survey report contained fabricated results. While there is value in broad surveying, such as one done for all faculty across an entire institution, doing so can miss aspects of satisfaction important or demotivating to specific populations of faculty. It would also be helpful to clearly communicate the intent of future surveying as well as better describe the survey methodology and analysis.

\section{Broader Surveying}

A recommendation for future research would be to take the findings from this study and develop a survey to be taken by biomedical research faculty at a subset of comparable institutions. This approach would be an opportunity to add validity to this study's findings in regards to their applicability to biomedical research faculty outside of this study's institution (i.e., a survey of endorsed concepts). If the findings from this study are not similar to those from biomedical faculty at other academic health centers, 
then it is likely these findings are specific to the one institution of this study

If the survey of endorsed concepts indicates that the results from this study are endorsed by biomedical research faculty at multiple academic health centers, the next step would be to conduct a broader national survey at academic health centers to gauge job satisfaction of biomedical research faculty. Large, quantitative surveys are often used to gauge job satisfaction (Babin Verret, 2012; Johnson, 2010) and surveying for endorsed concepts would minimize this study's initial concern that existing quantitative surveys do not always ask about issues important to the population. A larger, nation-wide survey could help organizations such as the National Institutes of Health, the National Science Foundation, or the American Association of Medical Colleges identify what issues to address and prioritize on a national scale in order to improve job satisfaction amongst the faculty who conduct their funded research projects.

\section{Female Faculty}

All female faculty in this study reported perceived differences in how they are treated as well as additional career difficulties when choosing to start a family. While faculty expressed that in some regards the current support mechanisms for female faculty and the treatment they receive from male colleagues are better than in the past, the difficulties particularly related to consistent external funding persist. Faculty of both genders described the constant need for funding as paramount for sustaining their careers, yet little support exists for female faculty to take any kind of break when starting a family. Future studies exploring how female faculty express this concern at other institutions is recommended in order to make recommendations for academic health centers to further support their female biomedical research faculty. 


\section{Soft Money Funding}

An additional area to further explore is the impact of soft money funding and faculty job satisfaction. While it is understood that soft money funds can lead to higher levels of stress due to the increasingly competitive landscape of federal grants (Holleman et al., 2014), less has been explored about how soft money further impacts satisfaction. Faculty in this study indicated that heavy reliance on soft money to fund one's salary and research can lead to distancing one's self from identifying with the institution. Therefore, it can be increasingly difficult for institutions to gather support or service if those faculty relying on soft money feel like independent contractors and not secure in their employment. Further research in this area would help to indicate at what point soft money decreases job satisfaction and how can institutions find balance between funding and faculty identification with the institution.

\section{Impact of Turnover on Remaining Faculty}

Finally, all faculty mentioned that having colleagues leave for other institutions is a demotivator that detracts from their overall job satisfaction. Because collegial relationships and collaboration was expressed as highly valued and something that adds great job satisfaction, it is important to know more about not just why faculty leave, but what happens to faculty who stay after their colleagues leave. Understanding this dynamic better would allow academic health centers to provide support mechanisms to faculty in disrupted workgroups that could potentially soften the impact of their colleague(s) leaving or perhaps even strengthen remaining relationships. Knowing more about this dynamic from talking to faculty after turnover occurs could be the start of what would eventually lead to recommendations for academic health centers. 


\section{Conclusion}

Once again, biomedical research faculty at this study's institution genuinely enjoy their jobs and prefer working at an academic health center. However, what detracts most from their satisfaction are issues that can largely be addressed institutionally. Academic health centers are the ideal environment and have all of the tools necessary for these faculty to find the next great advances in human health. However, more can be done to increase job satisfaction of biomedical research faculty. It would be impossible to expect all faculty to be entirely satisfied with their jobs all the time, but academic health centers can increase overall job satisfaction by addressing one issue at a time. 


\section{References}

AAHC. (2014). About AAHC. Retrieved March 10, 2016, from http://www.aahcdc.org/About.aspx

Antonio, A. L. (2003). Diverse student bodies, diverse faculties: The success or failure of ambitions to diversity faculty can depend on the diversity of student bodies. Academe, 89(6), 14-17.

Babin Verret, L. (2012). Factors affecting university STEM faculty job satisfaction. Louisiana State University and Agricultural and Mechanical College. Retrieved from http://etd.1su.edu/docs/available/etd-01202012195627/unrestricted/Lisa.Verret.pdf

Bateh, J. T. (2013). Leadership styles and faculty satisfaction in the state university system of Florida. Walden University.

Bender, K. A., \& Heywood, J. S. (2006). Job satisfaction of the highly educated: The role of gender, academic tenure, and earnings. Scottish Journal of Political Economy, 53(2), 253-279. Retrieved from http://10.0.4.87/j.1467-9485.2006.00379.x

Bilimoria, D., Perry, S. R., Liang, X., Stoller, E. P., Higgins, P., \& Taylor, C. (2006). How do female and male faculty members construct job satisfaction? The roles of perceived institutional leadership and mentoring and their mediating processes. The Journal of Technology Transfer, 31(3), 355-365. http://doi.org/10.1007/s10961006-7207-z

Bozeman, B., \& Gaughan, M. (2011). Job satisfaction among university faculty: Individual, work, and institutional determinants. Journal of Higher Education, 82(2), 154-186. Retrieved from http://dx.doi.org/10.1353/jhe.2011.0011 
Brannen, D., Bustos, R., Crute, T., Hardy, S., Lorenti, S., Mazzoli, A., ... PowellWilliams, M. (2014). Faculty morale survey report. Augusta, GA. Retrieved from http://gru.edu/universitysenate/senate/facultyrights/documents/fac_rights_respon_annual_rpt_2014.pdf

Brief, A. P. (1998). Attitudes in and around organizations. Thousand Oaks, CA: Sage Publications.

Callister, R. R. (2006). The impact of gender and department climate on job satisfaction and intentions to quit for faculty in science and engineering fields. The Journal of Technology Transfer, 31(3), 367-375. http://doi.org/10.1007/s10961-006-7208-y

Cohen, M. D., \& March, J. G. (2000). Leadership in an organized anarchy. In M. C. Brown II (Ed.), Organization \& governance in higher education (5th ed., pp. 1635). Boston: Pearson Custom Publishing.

Cole, T. R., Goodrich, T. J., \& Gritz, E. R. (2009). Faculty health in academic medicine. (T. R. Cole, T. J. Goodrich, \& E. R. Gritz, Eds.). Totowa, NJ: Humana Press. http://doi.org/10.1007/978-1-60327-451-7

Eyupoglu, S. Z., \& Saner, T. (2009). Job satisfaction: Does rank make a difference? African Journal of Business Management, 3(10), 609-615. http://doi.org/10.5897/AJBM09.157

Flaherty, C. (2016). Cluster-Hiring Cluster \&\%*\#? Retrieved from https://www.insidehighered.com/news/2016/02/01/uc-riverside-faculty-surveysuggests-outrage-cluster-hiring-initiative

Foster, M., \& Lyman, S. (2012). Faculty morale survey 2012 a biennial report. Retrieved from 
FACULTY MORALE IN BIOMEDICAL RESEARCH

http://www.missouristate.edu/assets/facultysenate/FacultyMoraleSurvey2012_Sum maryRepory_VOTE.pdf

Fox, M. F., \& Colatrella, C. (2006). Participation, performance, and advancement of women in academic science and engineering: What is at issue and why. The Journal of Technology Transfer, 31(3), 377-386. http://doi.org/10.1007/s10961-006-7209-x

Glesne, C. (2015). Becoming qualitative researchers: An introduction (5th ed.). Pearson.

Hagedorn, L. S. (2000). Conceptualizing faculty job satisfaction: Components, theories, and outcomes. In L. S. Hagedorn (Ed.), What contributes to job satisfaction among faculty and staff (pp. 5-20). San Francisco, CA: Jossey-Bass.

Herzberg, F., Mausner, B., \& Snyderman, B. B. (1959). The motivation to work. New York, NY: Wiley.

Holleman, W., Cofta-Woerpel, L. M., \& Gritz, E. R. (2014). Stress and morale of academic biomedical scientists. Academic Medicine : Journal of the Association of American Medical Colleges, 1-3. http://doi.org/10.1097/ACM.0000000000000533

Holleman, W., \& Gritz, E. R. (2013). Biomedical burnout. Nature, 500, 613-614.

Johnson, M. L. (2010). Significant factors influencing overall faculty satisfaction at public 2-year colleges. University of Phoenix.

Johnsrud, L. K., \& Rosser, V. J. (2002). Faculty members' morale and their intention to leave. The Journal of Higher Education, 73(4), 518-542.

Kezar, A. (2014). Women's contribution to higher education leadership and the road ahead. In K. A. Longman \& S. R. Madsen (Eds.), Women \& leadership in higher education (pp. 117-134). Information Age Publishing.

Kezar, A., \& Lester, J. (2008). Leadership in a divided world of feminism. Journal of 
Women in Higher Education, 1(1), 49-73.

Laden, B. V., \& Hagedorn, L. S. (2000). Job satisfaction among faculty of color in academe: Individual survivors or institutional transformers? In L. S. Hagedorn (Ed.), What contributes to job satisfaction among faculty and staff (pp. 57-66). San Francisco, CA: Jossey-Bass.

Lowi, T. J., Ginsberg, B., Shepsle, K. A., \& Ansolabehere, S. (2013). American government: Power and purpose (3rd ed.). New York, NY: W. W. Norton \& Company.

Marburger, J. H. I. (2015). Science policy up close. (R. P. Crease, Ed.). Cambridge, MA: Harvard University Press.

Marion, R., \& Gonzales, L. D. (2014). Leadership in education: Organizational theory for the practitioner (2nd ed.). Long Grove, IL: Waveland Press, Inc.

Maxwell, J. A. (2013). Qualitative research design: An interactive approach (3rd ed.). Thousand Oaks, CA: Sage Publications.

Mintzberg, H. (2000). The professional bureaucracy. In M. C. Brown III (Ed.), Organization \& governance in higher education (pp. 50-70). Boston: Pearson Custom Publishing.

Mitchell, M., \& Leachman, M. (2015). Years of cuts threaten to put college out of reach for more students. Retrieved May 15, 2015, from http://www.cbpp.org/research/state-budget-and-tax/years-of-cuts-threaten-to-putcollege-out-of-reach-for-more-students

Nyquist, J. G., Hitchcock, M. A., \& Teherani, A. (2000). Faculty satisfaction in academic medicine. In L. S. Hagedorn (Ed.), What contributes to job satisfaction among 
FACULTY MORALE IN BIOMEDICAL RESEARCH

faculty and staff (pp. 33-43). San Francisco, CA: Jossey-Bass.

Rice, R., \& Austin, A. (1988). High faculty morale: What exemplary colleges do right. Change: The Magazine of Higher Learning, 20(2), 50-58.

Ropers-Huilman, B. (2000). Aren't you satisfied yet? Women faculty members' interpretations of their academic work. In L. S. Hagedorn (Ed.), What contributes to job satisfaction among faculty and staff (pp. 21-32). San Francisco, CA: JosseyBass.

Rosser, V. J. (2004). Faculty members' intention to leave: A national study on their work life and satisfaction. Research in Higher Education, 45(3), 285-310.

Rosser, V. J. (2005). Measuring the change in faculty perceptions over time : An examination of their work life and satisfaction. Research in Higher Education, 46(1), 81-108. http://doi.org/10.1007/s

Sabharwal, M., \& Elizabeth, C. A. (2009). Faculty job satisfaction across gender and discipline. The Social Science Journal, 46, 539-556. Retrieved from http://cspo.org/legacy/library/100416F7SD_lib_SabharwalMandCor.pdf

Saldaña, J. (2016). The coding manual for qualitative researchers (3rd ed.). Thousand Oaks, CA: Sage Publications.

Seifert, T. A., \& Umbach, P. D. (2008). The effects of faculty demographic characteristics and disciplinary context on dimensions of job satisfaction. Research in Higher Education, 49(4), 357-381. Retrieved from http://dx.doi.org/10.1007/s11162-007-9084-1

Shockness, S. (2015). Faculty perspectives on factors impacting work as nurse educators. Walden University. 
Smart, J. C. (1990). A causal model of faculty turnover intentions. Research in Higher Education, 31(5), 405-424. http://doi.org/10.1007/BF00992710

Smolka, a. J., Halushka, P. V., \& Garrett-Mayer, E. (2015). The faculty costs to educate a biomedical sciences graduate student. Cell Biology Education, 14(1), 14:ar3,114:ar3,8. http://doi.org/10.1187/cbe.14-06-0106

Stein, D. G., \& Candler, A. G. (2007). Hard money \soft money: The two cultures. The Academic Exchange, 9(6).

TWU. (2015). Non-monetary approaches to improving faculty morale. Retrieved from https://www.twu.edu/downloads/senate/Nonmonetary.pdf

Wolf-Wendel, L., \& Ward, K. (2014). Academic mothers: Exploring disciplinary perspectives. Innovative Higher Education, 40(1), 19-35.

http://doi.org/10.1007/s10755-014-9293-4 


\section{Appendix A: Introduction Email}

Dear [Potential Participant Name],

My name is Rick Goranflo and I am a doctoral student at Portland State University. I am studying issues pertaining to morale and job satisfaction for faculty that conduct biomedical research. It is a topic especially important to me both academically and professionally.

I am reaching out to you as an invitation to participate in what will become my dissertation study. You specifically have been chosen due to your status as a tenure-track biomedical scientist at an academic health center. If you agree to participate, you will be asked to engage in an in-person interview that will last approximately one hour. The interview can take place wherever you would be most comfortable discussing issues pertaining to aspects of your career that impact your own personal morale and job satisfaction. These interviews will be audio recorded, transcribed, and you will be given a pseudonym so all responses are kept confidential. You may also receive a request for a follow up interview or short email questions if any clarification is needed at a later date.

Risks you may experience in this study are likely minimal. Most likely risks are feeling of anxiety, worrying, or reliving any previous negative experiences related to the questions you will be asked. You may also experience stress from disclosing information you would otherwise not want made public. If at any point you feel you do not want to 
answer a question or if you want to stop the interview, you may do so.

Benefits from participating in this study potentially include the opportunity to reflect on the positive things in your work environment and a reminder of what makes biomedical research important to you. The other benefit is adding to the academic literature information specifically about what impacts morale and job satisfaction to faculty working in your area. This knowledge can then potentially be used by academic health centers to assess their own issues regarding morale and job satisfaction and improve them.

Your participation in this study is completely voluntary and will not impact your status with your employer in any way. You may also withdraw at any time and have your responses removed from the study.

If you have questions at any time, please contact Rick Goranflo and goranflo@pdx.edu or 541-602-6024. Please keep a copy of this letter for your records.

If you agree to participate, please reply to this email with your intent to participate as well as answer a few basic demographic questions below.

Gender:

Ethnicity:

Current academic rank: 
FACULTY MORALE IN BIOMEDICAL RESEARCH

What year you began your academic career in research:

Number of academic institutions have you worked at:

How long you worked at your current institution:

Sincerely,

\author{
Rick Goranflo \\ EdD Student
}

Portland State University 
Appendix B: Informed Consent

The Portland State University

\title{
Consent to Participate in Research
}

\author{
Faculty Job Satisfaction and Morale in Biomedical Research
}

November 2016

\section{Introduction}

You are being asked to participate in a research study that is being done by Richard Goranflo, who is the Principal Investigator, from the Department of Educational Leadership, at Portland State University in Portland, Oregon. This research is studying morale and job satisfaction of faculty in biomedical research programs.

You are being asked to participate in this study because of your status as a tenure-track biomedical scientist at an academic health center.

This form will explain the research study, and will also explain the possible risks as well as the possible benefits to you. We encourage you to talk with your family and friends before you decide to take part in this research study. If you have any questions, please ask one of the study investigators.

\section{What will happen if I decide to participate?}

If you agree to participate, the following things will happen:

If you agree to participate, you will be asked to engage in an in-person interview that will last approximately one hour. The interview can take place wherever you would be most comfortable discussing issues pertaining to aspects of your career that impact your own personal morale and job satisfaction. These interviews will be audio recorded, transcribed, and you will be given a pseudonym so all responses are kept confidential. You may also receive a request for a follow up interview or short email questions if any clarification is needed at a later date.

\section{How long will I be in this study?}

Participation in this study will take a total of approximately one hour.

\section{What are the risks or side effects of being in this study?}

There are risks of stress, emotional distress, inconvenience and possible loss of privacy and confidentiality associated with participating in a research study.

Most likely risks are feeling of anxiety, worrying, or reliving any previous negative experiences related to the questions you will be asked. You may also experience stress from disclosing information you would 
otherwise not want made public. If at any point you feel you do not want to answer a question or if you want to stop the interview, you may do so.

For more information about risks and discomforts, ask the investigator.

\section{What are the benefits to being in this study?}

Benefits from participating in this study potentially include the opportunity to reflect on the positive things in your work environment and a reminder of what makes biomedical research important to you. The other benefit is adding to the academic literature information specifically about what impacts morale and job satisfaction to faculty working in your area. This knowledge can then potentially be used by academic health centers to assess their own issues regarding morale and job satisfaction and improve them.

\section{How will my information be kept confidential?}

We will take measures to protect the security of all your personal information, but we cannot guarantee confidentiality of all study data. Data gathered from this study will be entirely electronic and stored on Portland State University's secured Google Drive cloud storage. Your responses will be kept completely anonymous and any references to your responses will be assigned a pseudonym. After publication, data from this study will be destroyed after one year.

Information contained in your study records is used by study staff. The Portland State University Institutional Review Board (IRB) that oversees human subject research and/or other entities may be permitted to access your records, and there may be times when we are required by law to share your information. It is the investigator's legal obligation to report child abuse, child neglect, elder abuse, harm to self or others or any life-threatening situation to the appropriate authorities, and; therefore, your confidentiality will not be maintained.

Your name will not be used in any published reports about this study.

Will I be paid for taking part in this study?

No

\section{Can I stop being in the study once I begin?}

Your participation in this study is completely voluntary. You have the right to choose not to participate or to withdraw your participation at any point in this study without penalty or loss of benefits to which you are otherwise entitled.

\section{Whom can I call with questions or complaints about this study?}

If you have any questions, concerns or complaints at any time about the research study, Richard Goranflo will be glad to answer them at 541-602-6024. 


\section{Whom can I call with questions about my rights as a research participant?}

If you have questions regarding your rights as a research participant, you may call the PSU Office for Research Integrity at (503) $725-2227$ or 1(877) 480-4400. The ORI is the office that supports the PSU Institutional Review Board (IRB). The IRB is a group of people from PSU and the community who provide independent oversight of safety and ethical issues related to research involving human participants. For more information, you may also access the IRB website at https://sites.google.com/a/pdx.edu/research/integrity.

\section{CONSENT}

You are making a decision whether to participate in this study. Your signature below indicates that you have read the information provided (or the information was read to you). By signing this consent form, you are not waiving any of your legal rights as a research participant.

You have had an opportunity to ask questions and all questions have been answered to your satisfaction. By signing this consent form, you agree to participate in this study. A copy of this consent form will be provided to you.

Name of Adult Subject (print) Signature of Adult Subject Date

\section{INVESTIGATOR SIGNATURE}

This research study has been explained to the participant and all of his/her questions have been answered. The participant understands the information described in this consent form and freely consents to participate.

Richard Goranflo

Name of Investigator/ Research Team Member (type or print)

(Signature of Investigator/ Research Team Member)

Date 


\section{Appendix C: Interview Protocol}

Introduction: First, thank you for agreeing to take time out of your busy day to speak with me. As a reminder, I am interviewing you in my capacity as a doctoral student at Portland State University exploring issues pertaining to morale and job satisfaction for faculty in biomedical research programs. Everything you share with me will be kept confidential, your responses will be assigned a pseudonym, and you can decline to answer any question at any time. If you agree to those conditions, I will then begin my audio recorder to record this interview.

While I have a list of questions in front of me, I hope to keep this interview rather casual and more like a conversation. Please feel free to add anything else that comes to mind or you feel is important to share.

1. Think back to when you first discovered your interest in biomedical research. What initially interested you in this career and what motivates you now?

2. Describe what is different about working at an academic health center as a scientist compared to other environments.

3. Describe to me how you prioritize your work

4. Tell me about how you are recognized for your work.

Follow up: Does that recognition typically come from the institution, your 

community of peers, or others? Describe the recognition.

5. What impact has your gender had on your work?

6. Describe ways in which you feel your career choice has impacted your personal or family life.

7. Leadership and politics typically change over time at colleges and universities. Tell me about your relationship or perspective historically with the university's administration.

8. Similarly, tell me about your relationships you have with other faculty in your research area?

9. What is the most rewarding aspect or accomplishment you have made related to your work as a scientist?

10. Given the topic of this study is to explore issues pertaining to job satisfaction, is there anything else we haven't touched on that you think is important to know? 
Appendix D: Example of Coding

\begin{tabular}{|c|c|c|c|}
\hline Mediator & Excerpt & Value & Magnitude \\
\hline Achievement & $\begin{array}{l}\text { "So, scientific discoveries, some of the best } \\
\text { stuff we've done we're doing right now } \\
\text { because it's finally translating into something. } \\
\text { Which is you know most of us want } \\
\text { something to eventually reach a clinic. So, for } \\
\text { me, right now when everything seems to be } \\
\text { kind of down in terms of funding and the } \\
\text { environment, that's what keeps me going } \\
\text { thinking "this is some of the coolest stuff } \\
\text { we've ever done." It's really exciting and you } \\
\text { want to tell everyone about it. "' }\end{array}$ & YES & POS \\
\hline Recognition & $\begin{array}{l}\text { "Part of not getting recognition even though it } \\
\text { frustrates me is my own fault. I'm also not a, } \\
\text { I'm really horrible at self promotion. I hate } \\
\text { that part. And if you want to talk about what I } \\
\text { hate about my job, I hate the whole self } \\
\text { promotion and I think this field promotes and } \\
\text { encourages people with huge egos because it's } \\
\text { all about self promotion. And if you don't self } \\
\text { promote you don't get recognition. }\end{array}$ & YES & NEG \\
\hline Work itself & $\begin{array}{l}\text { "I'm absolutely still motivated by conducting } \\
\text { scientific research because if it weren't fun } \\
\text { and really enjoyable, it wouldn't be worth } \\
\text { some of the crap. When it stops being fun I } \\
\text { stop doing this." }\end{array}$ & YES & POS \\
\hline Responsibility & $\begin{array}{l}\text { "I have a lot of responsibility that comes with } \\
\text { autonomy. It is sort of having your own } \\
\text { business being a professor and having a lab. } \\
\text { You have to go out and raise the money for it } \\
\text { and I've spent a lot of years just trying to raise } \\
\text { the money, which is really hard, but also } \\
\text { really exciting when it comes together." }\end{array}$ & YES & POS \\
\hline Advancement & $\begin{array}{l}\text { "When you've been somewhere longer, it's } \\
\text { one of those things when the correlation is } \\
\text { messed up because you've been there longer } \\
\text { and probably have a higher academic rank so } \\
\text { the two are kind of confounded, but um I think } \\
\text { the sorts of things that I've been asked to do } \\
\text { have been more complex. Um, and probably } \\
\text { have more of an impact at a higher academic } \\
\text { rank. That's probably because I've been here }\end{array}$ & $\mathrm{NO}$ & $\mathrm{NEU}$ \\
\hline
\end{tabular}




\begin{tabular}{|c|c|c|c|}
\hline & longer though more so than rank." & & \\
\hline Salary & $\begin{array}{l}\text { "At one point we had a financial model where } \\
\text { we had bonuses and things like that and my } \\
\text { department and I were competitive with that, } \\
\text { but really what was more important was } \\
\text { having stability. What the new contract now } \\
\text { does is give you that stability rather than that } \\
\text { potential for bonuses. We're not in this for } \\
\text { the money. If we were we would all be in } \\
\text { industry." }\end{array}$ & $\mathrm{NO}$ & NEU \\
\hline Gender & $\begin{array}{l}\text { "There are definitely more women in } \\
\text { leadership positions and that's a good thing, } \\
\text { but if you look at a lot of the women in power } \\
\text { on this campus, that doesn't matter to them. } \\
\text { And that's not how they got there. And } \\
\text { unfortunately a lot of women in power in } \\
\text { science are women who have male attributes." }\end{array}$ & YES & MIX \\
\hline Institutional type & $\begin{array}{l}\text { "Since coming here I think I probably didn't } \\
\text { even appreciate the resources that I would } \\
\text { have as a result of being at a health center. } \\
\text { The way I conduct my research now is quite } \\
\text { different than how my previous bosses did." }\end{array}$ & YES & POS \\
\hline $\begin{array}{l}\text { Academic } \\
\text { discipline }\end{array}$ & $\begin{array}{l}\text { "What initially drove me was a love of math } \\
\text { and a love of things logical. I love figuring } \\
\text { things out. I still love puzzles and problems } \\
\text { and things like that. I like figuring out things. } \\
\text { In research I really just love problem solving } \\
\text { and troubleshooting. Figuring out what's } \\
\text { going wrong with your experiment and trying } \\
\text { to figure out what it is and how to get around } \\
\text { it. What also helps me is I really like having a } \\
\text { larger goal to help people. Sometimes in your } \\
\text { day to day work it's very frustrating, but if } \\
\text { you have that larger goal that the knowledge } \\
\text { that you're gaining or the material that you're } \\
\text { working on could some day help people, that } \\
\text { kind of sustains me and my research." }\end{array}$ & YES & POS \\
\hline $\begin{array}{l}\text { Collegial } \\
\text { relationships }\end{array}$ & $\begin{array}{l}\text { "The real strength (here) is the collaboration } \\
\text { with other faculty. Despite all of this other } \\
\text { noise, people really enjoy getting together to } \\
\text { talk about science and share ideas and } \\
\text { participating." }\end{array}$ & YES & POS \\
\hline $\begin{array}{l}\text { Student quality } \\
\text { or relationships }\end{array}$ & $\begin{array}{l}\text { "Teaching is part of the reason I'm here. It's } \\
\text { one of the ways we promote the field and }\end{array}$ & YES & POS \\
\hline
\end{tabular}




\begin{tabular}{|c|c|c|c|}
\hline & $\begin{array}{l}\text { advance young scientists. I like teaching and } \\
\text { put a lot of work into it. Most faculty } \\
\text { participate in graduate programs as a } \\
\text { volunteer because it makes no difference in } \\
\text { your salary. Most people do it because they } \\
\text { think it's an important part of the mission. } \\
\text { There's a certain element of idealism to that." }\end{array}$ & & \\
\hline Administration & $\begin{array}{l}\text { "My enjoyment has greatly diminished over } \\
\text { time by the ridiculous burdens of } \\
\text { administration and the terrible landscape of } \\
\text { grant funding. I think many people in my } \\
\text { position are weighing that balance and losing } \\
\text { that balance is a terrible blow." }\end{array}$ & $\mathrm{NO}$ & NEG \\
\hline $\begin{array}{l}\text { Institutional } \\
\text { climate or } \\
\text { culture }\end{array}$ & $\begin{array}{l}\text { "It has been said this university lacks the } \\
\text { tradition of being a university and that's the } \\
\text { source of many woes. There is no } \\
\text { administrative understanding of being a } \\
\text { university, but we're a biomedical corporation } \\
\text { or a hospital corporation with schools tacked } \\
\text { onto the side. I see no reason to see why } \\
\text { that's going to change." }\end{array}$ & $\mathrm{NO}$ & NEG \\
\hline
\end{tabular}

